\title{
The Chirality of Isotopomers of Glycine Compared using Next-Generation QTAIM
}

\author{
Xing $\mathrm{Nie}^{1}$, Yong Yang ${ }^{1}$, Tianlv Xu${ }^{1}$, Malgorzata Biczysko ${ }^{2}$, Steven Kirk ${ }^{1}$, and Samantha \\ Jenkins ${ }^{1}$ \\ ${ }^{1}$ Hunan Normal University \\ ${ }^{2}$ Shanghai University
}

December 8, 2021

\begin{abstract}
The effect of the presence of a deuterium (D) or tritium $(\mathrm{T})$ isotope bonded to the alpha carbon of glycine is determined without the need to apply external forces e.g. electric fields or using normal mode analysis. Isotopic effects were accounted for using the mass-dependent diagonal Born-Oppenheimer energy correction (DBOC) at the CCSD level of theory. We calculated the stress tensor trajectories of the dominant $\mathrm{C}-\mathrm{N}$ bond within next generation quantum theory of atoms in molecules (NG-QTAIM). S-character chirality was discovered using the stress tensor trajectories, instead of the Cahn-Ingold-Prelog (CIP) rules, for ordinary glycine. The S-character chirality was preserved after the substitution of the $\mathrm{H}$ on the alpha carbon for a $\mathrm{D}$ isotope but transformed to R-character chirality after replacement with the $\mathrm{T}$ isotope. This reversal of the chirality depending on the presence of a single $\mathrm{D}$ or $\mathrm{T}$ isotope bound to the alpha carbon adds to the debate on the nature of the extraterrestrial origins of chirality in simple amino acids. We demonstrate that NG-QTAIM is a promising tool for understanding isotopic induced electronic charge density changes, useful in analysis of infrared (IR) or circular dichroism (CD) spectra explaining changes in mode couplings and bands intensities or sign.
\end{abstract}

\section{The Chirality of Isotopomers of Glycine Compared using Next-Generation QTAIM}

Xing $\mathrm{Nie}^{1}$, Yong Yang ${ }^{1}$, Tianlv $\mathrm{Xu}^{1}$, Malgorzata Biczysko ${ }^{2}$, Steven R. Kirk ${ }^{1^{*}}$ and Samantha Jenkins $^{1^{*}}$

${ }^{1}$ Key Laboratory of Chemical Biology and Traditional Chinese Medicine Research and Key Laboratory of Resource National and Local Joint Engineering Laboratory for New Petro-chemical Materials and Fine Utilization of Resources, College of Chemistry and Chemical Engineering, Hunan Normal University, Changsha, Hunan 410081, China; niexing0623@foxmail.com (X N); yy0801@foxmail.com (Y Y); xutl@hunnu.edu.cn (T $X)$

${ }^{2}$ International Centre for Quantum and Molecular Structures,1/F Building E,Shanghai University, 99 Shangda Road, Shanghai, 200444 China

email: samanthajsuman@gmail.com

email: steven.kirk@cantab.net

The effect of the presence of a deuterium (D) or tritium (T) isotope bonded to the alpha carbon of glycine is determined without the need to apply external forces e.g. electric fields or using normal mode analysis. Isotopic effects were accounted for using the mass-dependent diagonal Born-Oppenheimer energy correction (DBOC) at the CCSD level of theory. We calculated the stress tensor trajectories of the dominant C-N bond within next generation quantum theory of atoms in molecules (NG-QTAIM). S-character chirality was 
discovered using the stress tensor trajectories, instead of the Cahn-Ingold-Prelog (CIP) rules, for ordinary glycine. The S-character chirality was preserved after the substitution of the $\mathrm{H}$ on the alpha carbon for a $\mathrm{D}$ isotope but transformed to R-character chirality after replacement with the $\mathrm{T}$ isotope. This reversal of the chirality depending on the presence of a single $\mathrm{D}$ or $\mathrm{T}$ isotope bound to the alpha carbon adds to the debate on the nature of the extraterrestrial origins of chirality in simple amino acids. We demonstrate that NG-QTAIM is a promising tool for understanding isotopic induced electronic charge density changes, useful in analysis of infrared (IR) or circular dichroism (CD) spectra explaining changes in mode couplings and bands intensities or sign.

\section{Introduction}

Isotopes, homo-electronic nuclei, showing distinct properties due to the different nuclear spins ${ }^{1,2}$, are omnipresent in the universe ${ }^{3}$. Traditionally, different properties have been associated with the mass difference and observed for instance in the variation of rate or equilibrium constant of two reactions (i.e. kinetic isotope effect or a thermodynamic isotope effect ${ }^{4}$. In principle, isotopes can occur independent of mass and spin because of symmetry restrictions on the molecular wavefunction leading to different symmetry selection rules for different isotopomers ${ }^{5}$. In this work we refer to the isotopic effect as a change of any property between molecules with distinct isotopic components (isotopomers). Nevertheless, the isotopic effects related to the lightest elements are most pronounced. The natural abundance of atomic isotopes is then reflected in the isotopic composition of molecules, but the artificial isotopic substitution- mainly of hydrogen $(\mathrm{H})$ to deuterium (D) has been used in many applications. One group of applications is related to the identification of species, or elucidation of molecular structures, by enhancing the amount of available experimental data (in mass spectrometry, optical spectroscopies, nuclear magnetic resonance (NMR) spectroscopy $)^{6-8}$. Alternatively, isotopic labeling allows one to mark and distinguish between specific parts of molecular systems, for instance in biomolecular chemistry and deuterium labeling for reaction analysis ${ }^{9-15}$. Among the isotopic substitutions of particular interest are those which change formally achiral molecules into chiral ones. Such newly created species can then be recognized by chiroptical spectroscopies, while Quack and co-workers reported the first quantitative investigations of a ground-state energy difference for the enantiomers of molecules that are chiral only by isotopic substitution ${ }^{16}$.

The origins of chiral molecules in space and its relation with the chiral asymmetry of life on the Earth still remains a mystery ${ }^{17}$. So far the only chiral molecule detected in the interstellar medium (ISM) is methyloxirane, however as it was identified by means of achiral (rotational) signals its enantiomeric composition is unknown $^{18}$. D and L forms of amino acids have been found in meteorites and comets, but L-enantiomeric excesses have been also established ${ }^{17}$. As the star-planet formation regions are characterized by very high $\mathrm{D} / \mathrm{H}$ ratio, contributing significantly to the formation of heavier molecules ${ }^{19}$, isotopically induced chirality maybe highly relevant in an astrochemical context. Oba et. al . reported experimental evidence of chiral glycine formation by the surface reaction of normal glycine in solid form with deuterium atoms at conditions simulating interstellar molecular clouds ${ }^{20}$. Chiral glycine is a known chiral trigger for amplifying an enantiomeric excess under certain conditions ${ }^{21}$ : these results add to the long standing debate on the origin of homochirality. The investigation of Kawasakiet. al . discovered that achiral meteoritic amino acids that included glycine with hydrogen isotope $(\mathrm{D} / \mathrm{H})$ chirality were the source of chirality in asymmetric autocatalysis with amplification of enantiomeric excess to enable the creation of highly enantioenriched 5-pyrimidyl alkanols ${ }^{21}$. Kawasaki et. al found that the chirality of the $\mathrm{S}$ and $\mathrm{R}$ enantiomers was mainly attributable to the very small difference between the C-D and C-H bond-lengths associated with the alpha carbon of glycine $^{21-23}$. The development of a highly sensitive method for the detection of isotopic chirality in meteoritic organic compounds such as amino acids ${ }^{24}$ with an achiral framework and isotopic enrichment therefore remains a challenging subject ${ }^{25}$.

The unknown chirality-helicity equivalence, that associates chirality with a helical characteristic, was recently located although not quantified by some of the current authors ${ }^{26}$ and was used to distinguish the S and R stereoisomers of lactic acid in agreement with the naming schemes from optical experiments. Some of the current authors have very recently published the derivation of the chirality-helicity equivalence that quantifies 
the chiral character in formally achiral species ${ }^{27}$. Consistency with our work was found from experiments by Beaulieu et al. on neutral molecules ${ }^{28}$ that utilize coherent helical motion of bound electrons. Abstract chirality measures for non-rigid objects have been used to associate optical rotation with the structure of a molecule ${ }^{29}$.

Within the Born-Oppenheimer (B.O.) approximation the electronic spectra of isotopomers are identical because the electronic Hamiltonian $\hat{H}_{e}$ in the BO approximation is not dependent on nuclear masses despite differences in their nuclei dynamics. Therefore isotope sensitivity is typically achieved via nuclear dynamics, for example by considering vibrational spectra. In this contribution we explore the effect of non-adiabatic coupling corrections and find that despite subtle differences that they induce in geometries those differences are sufficient to be reliably captured by non-scalar QTAIM quantities.

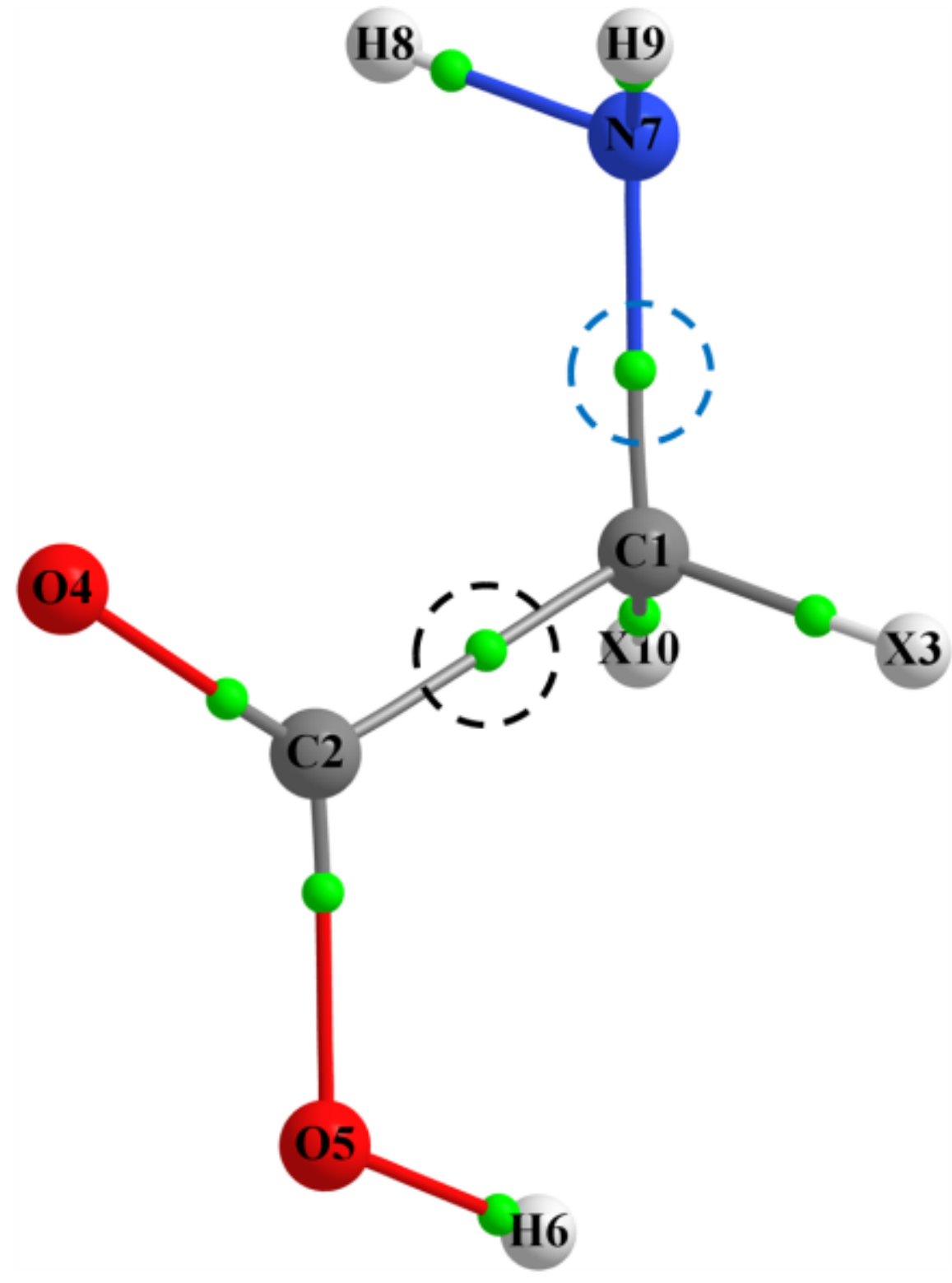

Scheme 1. The molecular graph of the singly deuterated and triterated isotopomers substituted onto the 
(alpha carbon) $\mathrm{C} 1$ atom of glycine: $\mathrm{S}_{\mathrm{a}}(\mathrm{X} 3=\mathrm{D} 3 / \mathrm{T} 3, \mathrm{X} 10=\mathrm{H} 10)$ (left panel) and $\mathrm{R}_{\mathrm{a}}(\mathrm{X} 3=\mathrm{H} 3, \mathrm{X} 10=$ $\mathrm{D} 10 / \mathrm{T} 10)$ (right panel). The notation $\mathrm{HH}$ will be used to denote $\mathrm{X} 3=\mathrm{H} 3, \mathrm{X} 10=\mathrm{H} 10$. The following notation HD is used for $\left\{\mathrm{S}_{\mathrm{a}}(\mathrm{X} 3=\mathrm{D} 3, \mathrm{X} 10=\mathrm{H} 10), \mathrm{R}_{\mathrm{a}}(\mathrm{X} 3=\mathrm{H} 3, \mathrm{X} 10=\mathrm{D} 10)\right\}$ and HT is denotes $\left\{\mathrm{S}_{\mathrm{a}}\right.$ $\left.(\mathrm{X} 3=\mathrm{T} 3, \mathrm{X} 10=\mathrm{H} 10), \mathrm{R}_{\mathrm{a}}(\mathrm{X} 3=\mathrm{H} 3, \mathrm{X} 10=\mathrm{T} 10)\right\}$. The torsional $\mathrm{C} 1-\mathrm{C} 2 B C P$ and torsional $\mathrm{C} 1-\mathrm{N} 7 B C P$ are indicated by the black and blue circles respectively, where the undecorated green spheres indicated the locations of bond critical points (BCPs).

In this article, we thus explore the extent to which subtle differences between the electronic structures of isotopomers can be captured by directly analyzing the electron density rather than by referring to the vibrational spectrum. Tritium is a spin- $\frac{1}{2}$ isotope of hydrogen, with effectively the same chemical shifts but with slightly higher sensitivity, dispersion and coupling constants ${ }^{30}$.

We consider the total electronic charge density because it is an observable ${ }^{31-33}$, rather than the wavefunction which is not. Total electronic charge density distributions are available even when sharp spectral lines are not such as is the case for the solid state. We will analyze density-dependent quantities of the deuterium and tritium isotopomers of glycine using QTAIM (Quantum Theory of Atoms in Molecules) ${ }^{34}$ and more specifically the directional next generation QTAIM see Scheme 1. Previously, the stress tensor trajectories $\mathrm{T}(s)$, within Next generation QTAIM, were used to quantify the O-H bond-flexing, bond-torsion and bond-axiality (formerly referred to as bond anharmonicity) contributions to be compared for different isotopomers of normal modes of the water molecule ${ }^{35}$. This earlier analysis enabled the coupling of intramolecular vibrational modes to be assessed and compared, particularly between the bending normal mode and the symmetric-stretch normal modes. The glycine conformer selected for current investigation is characterized by an intense IR band ${ }^{36}$ involving the $\mathrm{C} 2-\mathrm{O} 5-\mathrm{H} 6$ bending coupled with the wagging vibration of methylene group $(\mathrm{C} 1 \mathrm{X} 3 \mathrm{X} 10)^{37}$, which is expected to be shifted by $\mathrm{D} / \mathrm{T}$ isotopic substitution at that alpha carbon (C1), see Scheme 1 . The ability to capture the chiral character and its changes upon the D/T substitution allows the direct analysis of differences in the chiroptical spectra. Such changes include sign changes, without the need to introduce approximations based on the normal modes description ${ }^{38}$.

\section{Theoretical Background}

The interested reader is referred to the complete mathematical expressions and explanations in the Supplementary Materials S1. QTAIM analysis ${ }^{39-45}$ is used to identifycritical points in the total electronic charge density distribution $\rho(\mathbf{r})$ where the gradient vector field [?] $\rho(\mathbf{r})=0$. There are four distinct categories of critical points according to the set of ordered eigenvalues $\lambda_{1}<\lambda_{2}<\lambda_{3}$, with the associated set of eigenvectors $\left(e_{\mathbf{1}}, e_{\mathbf{2}}, e_{\mathbf{3}}\right)$, of the Hessian matrix of the electronic charge density, $\rho(\mathbf{r})$, defined as the matrix of partial second derivatives with respect to the spatial coordinates, [?]:[?] $(\mathbf{r})$. Critical points are labeled using the notation $(R, \omega)$ where $R$ is the rank of the Hessian matrix and $\omega$ is the signature; the $(3$, -3) [nuclear critical point $(N C P)$, a local maximum generally corresponding to a nuclear location], $(3,-1)$ and $(3,1)$ [saddle points, called bond critical points $(B C P)$ and ring critical points $(R C P)$, respectively] and $(3,3)$ [the cage critical points $(C C P)]$. In this investigation we will only be considering bond critical points $(B C P \mathrm{~s})$.

The ellipticity $\varepsilon$, quantifies the relative accumulation of $\rho\left(\mathbf{r}_{\mathbf{b}}\right)$ in the two directions $\left(e_{\mathbf{1}}\right.$ and $\left.e_{\mathbf{2}}\right)$ perpendicular to the bond-path at $r_{b}$. For ellipticity values $>0$, the associated $\lambda_{1}$ and $\lambda_{2}$ Hessian eigenvalues correspond to the shortest and longest axes of the elliptical distribution of $\rho\left(\mathbf{r}_{\mathbf{b}}\right)$, respectively.

Bond-flexing distortions involve the stretching of a bond (bond-path) so that the bond-path length (BPL) exceeds the bonded inter-nuclear geometric separation distance. A shift of a $B C P$ position along the containing bond-path due to changes to bonded inter-nuclear separations results in the presence of $B C P$ sliding. As a consequence of this $B C P$ sliding the chemical nature of the bond is dependent on the relative position of the $B C P$, i.e. we can quantify a degree of bond-axiality ${ }^{35}$. The construction of the stress tensor trajectories $\mathrm{T}_{\sigma}(\mathrm{s})$ involves the required additional symmetry breaking to identify chirality in the form of the $e_{\mathbf{3} \sigma}$ eigenvector. This enables the $\mathrm{T}_{\sigma}(\mathrm{s})$ corresponding to the counterclockwise $(\mathrm{CCW})$ and clockwise $(\mathrm{CW})$ directions of torsion to be distinguished even for the highly symmetrically positioned torsional C1-C2 $B C P$ 
, see Scheme 1 . To be consistent with optical experiments as previously undertaken ${ }^{46}$ we define $\boldsymbol{\Sigma}_{\boldsymbol{\sigma}}$ (lefthanded) character to be dominant over $\mathbf{R}_{\sigma}$ character (right-handed) for values of the chirality $\mathrm{C}_{\sigma}(\mathrm{CCW})>$ (CW) since CCW and CW represent left and right handed directions of torsion respectively. Note the use of the subscript " $\sigma$ " because we are using the stress tensor $T_{\sigma}(\mathrm{s})$ in the stress tensor $\mathrm{U}_{\sigma}$-space. The chirality $\mathrm{C}_{\sigma}$ of a torsion bond (in this work the torsional C1-C2 BCP and torsional C1-N7BCP ) is defined by the difference in the maximum $\mathrm{T}_{\sigma}(s)$ projections (the dot product of the stress tensor $\boldsymbol{\varepsilon}_{1 \sigma}$ eigenvector and the $B C P$ shiftdr $)$ of the $\mathrm{T}_{\sigma}(\mathrm{s})$ values between the $\mathrm{CCW}$ and $\mathrm{CW}$ torsions:

$\mathrm{C}_{\sigma}=\left[\left(\varepsilon_{1 \sigma}[;] \delta \rho\right)_{\mu \alpha \xi}\right]_{\mathbf{C C W}^{-}}\left[\left(\varepsilon_{1 \sigma}[;] \delta \rho\right)_{\mu \alpha \xi}\right]_{\mathbf{C W}}(1)$

These torsions correspond to the CW $\left(-180.0^{\circ}[?] \vartheta[?] 0.0^{\circ}\right)$ and $\operatorname{CCW}\left(0^{\circ}\right.$ [?] $\left.\vartheta[?] 180.0^{\circ}\right)$ directions of the torsion $\vartheta$. The chirality $\mathrm{C}_{\sigma}$ quantifies the bond torsion direction $\mathrm{CCW}$ vs. $\mathrm{CW}$, i.e. circularmotion, since $\boldsymbol{\varepsilon}_{1 \boldsymbol{\sigma}}$ is the most preferred direction of charge density $\rho\left(\mathbf{r}_{\mathbf{b}}\right)$ accumulation.

The response of the $\mathrm{C}-\mathrm{H} / \mathrm{D} / \mathrm{T}$ bonds to the $\mathrm{CCW}$ vs. $\mathrm{CW}$ torsions uses equation $\mathbf{1}(\mathbf{a})$ but does not define a chirality $\mathrm{C}_{\sigma}$ associated with a torsional bond of a molecule. Instead the response is referred to as the bond-twist $\mathrm{T}_{\sigma}$

The least preferred $\left(\boldsymbol{\varepsilon}_{\mathbf{2} \boldsymbol{\sigma}}\right)$ direction of $\rho\left(\mathbf{r}_{\mathbf{b}}\right)$ corresponds to a more 'difficult' bond distortion than bond torsion, that we refer to as the bond-flexing $F_{\sigma}$ that is defined as:

$F_{\sigma}=\left[\left(\varepsilon_{2 \sigma}[;] \delta \rho\right)_{\mu \alpha \xi}\right]_{C C W^{-}}\left[\left(\varepsilon_{2 \sigma}[;] \delta \rho\right)_{\mu \alpha \xi}\right]_{C W}$

The bond-axiality $\mathrm{A}_{\sigma}$ quantifies the resultant $B C P$ motion along the bond-path, that coincides with the $\boldsymbol{\varepsilon}_{\mathbf{3}}$ direction, that we refer to as the bond-axial direction. The bond-axiality $\mathrm{A}_{\sigma}$ provides a measure of symmetry breaking ${ }^{35}$ in response to the bond torsion directions (CCW vs. CW). The bond-axiality $\mathrm{A}_{\sigma}$ is defined as:

$\mathrm{A}_{\sigma}=\left[\left(\varepsilon_{3 \sigma}[;] \delta \rho\right)_{\mu \alpha \xi}\right]_{\mathrm{CCW}^{-}}\left[\left(\varepsilon_{3 \sigma}[;] \delta \rho\right)_{\mu \alpha \xi}\right]_{\mathrm{CW}}$

The sign $( \pm)$ of the chirality $\mathrm{C}_{\sigma}$, bond twist $\mathrm{T}_{\sigma}$ and bond-axiality $\mathrm{A}_{\sigma}$ determines the dominance of $\boldsymbol{\Sigma}_{\boldsymbol{\sigma}}\left(\mathrm{C}_{\sigma}>\right.$ $0)$ and $\mathbf{P}_{\sigma}\left(\mathrm{C}_{\sigma}<0\right)$ character. A high degree of bond-axiality $\mathrm{A}_{\sigma}$ corresponds to a large difference in the value of the $\left\{\epsilon_{3 \sigma} . \delta \rho\right\}_{\mu \alpha \xi}$ for the CCW and CW torsion directions.

The stereoisomeric excess $X_{\sigma}$ is defined as the ratio of the magnitude of the $C_{\sigma}$ values of the $S_{a}$ and $R_{a}$ stereoisomers of the torsional C1-N7 BCPand represents the Next generation QTAIM interpretation of the enantiomeric excess.

\section{Computational Details}

Isotopically substituted and unsubstituted molecular structures were first geometry-optimized to an energy minimum. The 6-311(2d,3p) basis set was selected and was used consistently in the geometry optimizations and all subsequent single-point calculations. Isotopic effects were included by performing the geometry optimization using a finite-difference approach using energies alone, calculated with the mass-dependent diagonal Born-Oppenheimer energy correction (DBOC) ${ }^{47,48}$ applied. This was carried out at the CCSD level of theory using the CFOUR code ${ }^{49}$, with the following tight convergence criteria (in atomic units): Maximum coupled-cluster amplitude change $>10^{-10}$, RMS energy gradient $<10^{-5}$, smallest linear equation (DIIS) residual $<10^{-10}$ and integrals tolerance $10^{-15}$.

Torsional scans (using constrained geometry optimization) were then made based on the converged structures from the previous step, with the $\mathrm{C} 1$ atom position coordinates and the $\mathrm{C} 1-(\mathrm{H}, \mathrm{D}, \mathrm{T}) 3$ and $\mathrm{C} 1-(\mathrm{H}, \mathrm{D}, \mathrm{T}) 10$ bond lengths constrained to their previously converged energy minimum values, using the B3LYP DFT functional, very tight geometry convergence criteria and an 'ultrafine' grid for the DFT integrals as implemented in the G09 vE. $01^{50}$ code. The full optimization Hessian was recalculated at every optimization step. Finally, single-point wavefunction calculations were run using G09, the same DFT functional, basis set and 
integral parameters, with the SCF convergence criteria $<10^{-10}$ RMS change in the density matrix, and the corresponding wavefunctions analysed using AIMAll ${ }^{51}$. Next generation QTAIM analyses for $\mathrm{U}_{\sigma}$-space trajectories and derived quantities were calculated from the resulting molecular graphs and wavefunctions using our in-house developed software package QuantVec (formerly AIMPAC2-Suite) ${ }^{52}$.

\section{Results and Discussion}

\subsection{The insufficiency of scalar measures for determining chirality and distinguishing stereoisomers}

Note we use the subscript "a" for the $\mathrm{S}$ and $\mathrm{R}$ stereoisomers of glycine because glycine is formally achiral and the stereoisomers are produced by the symmetry breaking effects of the introduction of the $\mathrm{D}$ and $\mathrm{T}$ isotopomers to the alpha carbon $(\mathrm{C} 1)$, where the CIP rules were used to allocate the $\mathrm{S}_{\mathrm{a}}$ and $\mathrm{R}_{\mathrm{a}}$ designations, see Scheme 1 . The variation of the (scalar) energies [?]E, BPL and ellipticity values $\varepsilon$ relative to $\vartheta=$ $0.0^{\circ}$, corresponding to the relaxed geometry of glycine, of the CW $\left(-180.0^{\mathrm{o}}[?] \vartheta[?] \quad 0.0^{\mathrm{o}}\right)$ and $\mathrm{CCW}\left(0.0^{\mathrm{o}}\right.$ [?] $\left.\vartheta[?] 180.0^{\circ}\right)$ torsions associated with the C1-C2 BCP for the pure glycine and singly deuterated and triterated glycine do not distinguish the isotopomers, seeFigure $\mathbf{1}(\mathbf{a}-\mathbf{b})$. The corresponding results for the C1-N7BCP display a high degree of asymmetry with respect to the torsion $\vartheta$, but do not distinguish between the isotopomers, seeFigure 1(c-d). The variation of the energies [?] $E$ relative to $\vartheta=0.0^{\underline{0}}$, associated with the $\mathrm{C} 1-\mathrm{N} 7 B C P$ and $\mathrm{C} 1-\mathrm{C} 2 B C P$, demonstrates the greater strength of interaction of the C1-N7 $B C P$ compared to the C1-C2 $B C P$, compare the left panels of Figure 1(a-b) with those of Figure $\mathbf{1}(\mathbf{c}-\mathbf{d})$. These include the distance measures for a torsion $\vartheta=0.0^{\circ}$ that indicate that the C1-N7 $B C P$ is closer to the $\mathrm{C} 1$ atom $(1.198$ a.u.) than the $\mathrm{C} 1-\mathrm{C} 2 \mathrm{BCP}$ (1.393 a.u.). This asymmetry in the location of the $B C P$ along the C1-N7 $B C P$ bond-path leads to an asymmetry of the variation of the BPL, see the middle panels of Figure 1(c-d) and the $B C P$ ellipticity $\varepsilon$, see the rights panels of Figure 1(c-d) with the CCW and CW torsions. In particular, the lower values of the C1-N7 BCP ellipticity $\varepsilon$ for the CCW $\left(0.0^{\mathrm{O}}\right.$ [?] $\vartheta$ [?] 180.0 $\left.0^{\mathrm{o}}\right)$ torsion compared to the CW $\left(-180.0^{\mathrm{O}}\right.$ [?] $\left.\vartheta[?] \quad 0.0^{\mathrm{O}}\right)$ torsion indicate a preference for the CCW torsion over the $\mathrm{CW}$ torsion. This is because a bond with lower ellipticity $\varepsilon$ values, e.g. single bonds, will undergo a torsion deformation more readily than bonds with a higher ellipticity $\varepsilon$ value, as is the case for double bonds. The symmetrical location of the C1-C2 BCP mid-way along the associated bond-path results in symmetrical variations of the BPL and the ellipticity $\varepsilon$ with the CCW and CW torsions and hence no preferred direction of torsion, $\mathrm{CCW}$ or $\mathrm{CW}$, is indicated.

Considering the limitations of the scalar $B C P$ ellipticity $\varepsilon$ in determining preferences of CCW over $\mathrm{CW}$ for the $\mathrm{C} 1-\mathrm{C} 2 \mathrm{BCP}$, we now proceed to examine the directional differences between the $\mathrm{CCW}$ and $\mathrm{CW}$ torsions of the torsional C1-N7 $B C P$ and C1-C2 $B C P$ with the vector-based stress tensor trajectories $\mathrm{T}_{\sigma}(\mathrm{s})$, seeFigure 1(c) and Figure 1(d) respectively. The $B C P$ ellipticity $\varepsilon$ does not take into account the bondpath eigenvector $\left(e_{\mathbf{3} \sigma}\right)$ and therefore cannot quantify torsional CCW vs. CW preferences for a symmetrically positioned C1-C2 $B C P$, see Figure 1(a-d) . 

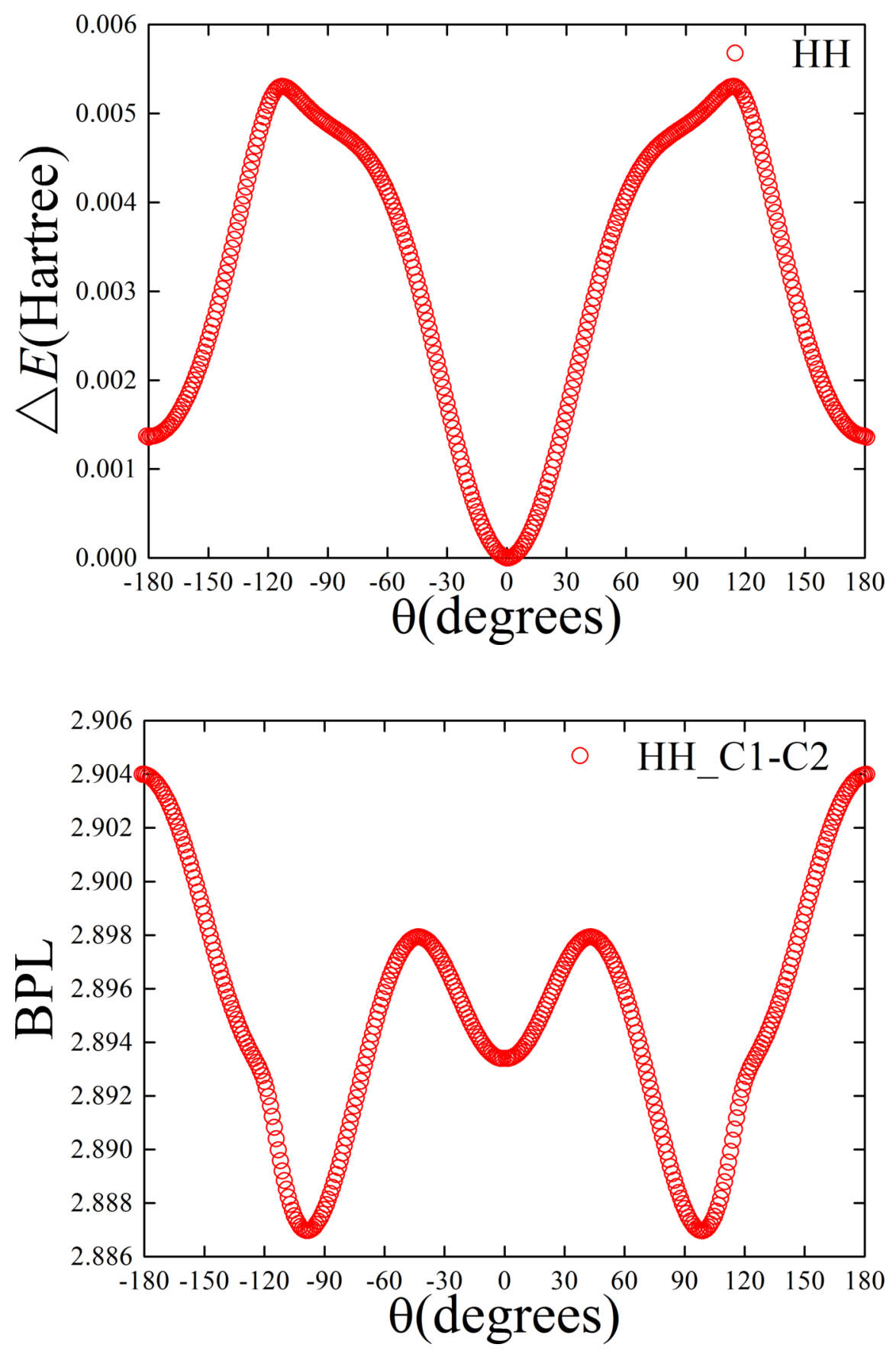


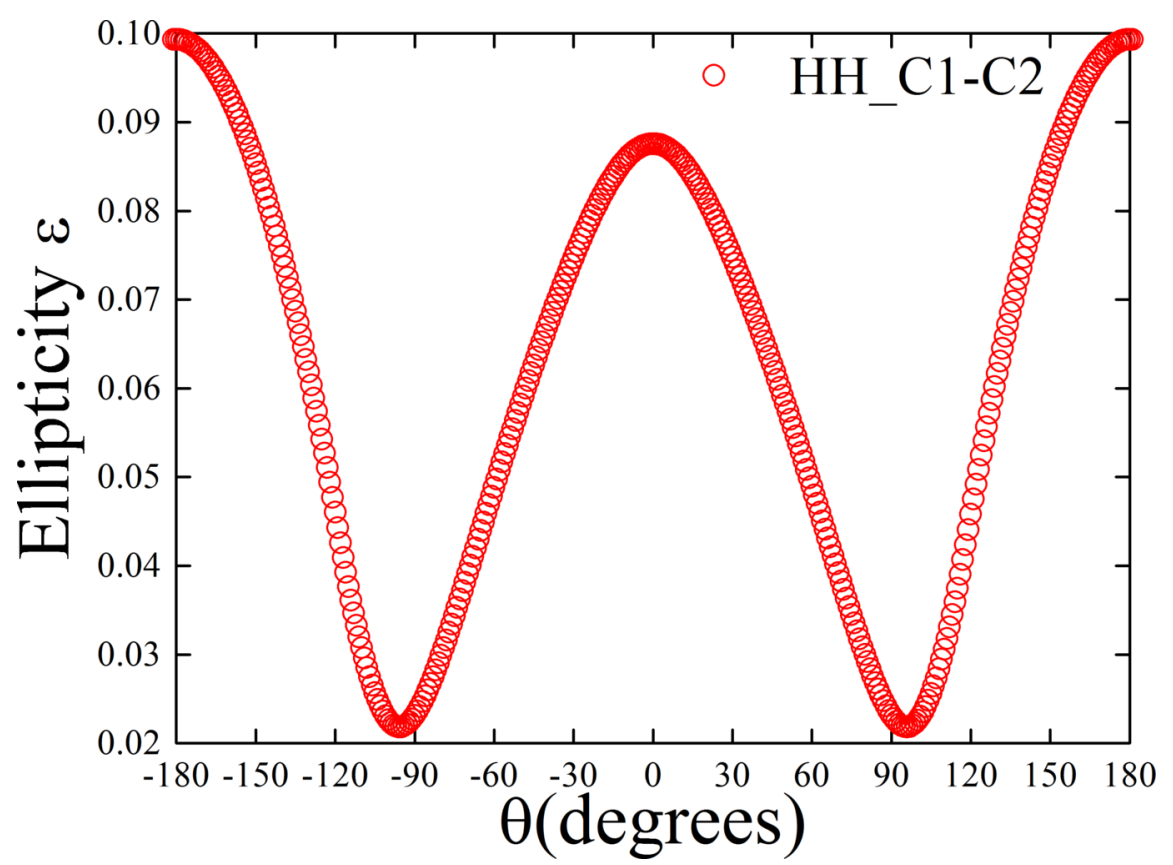

(a)

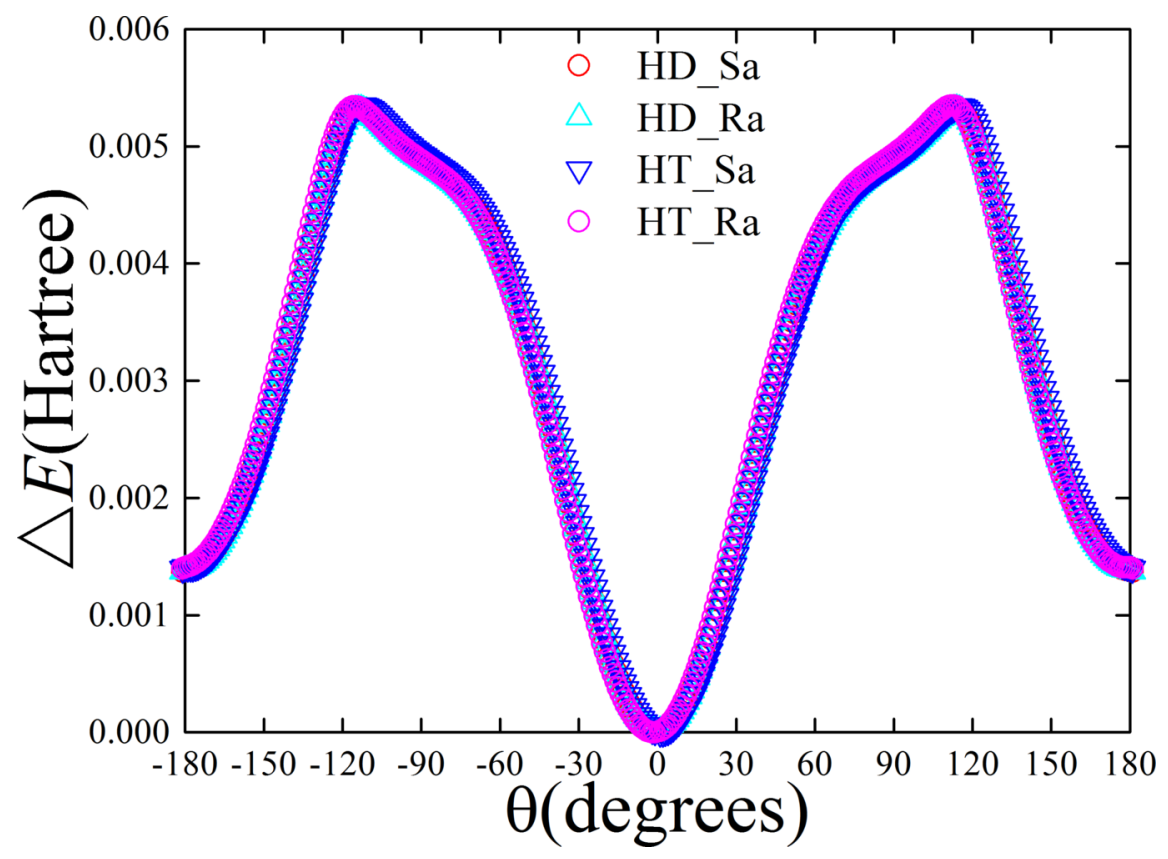



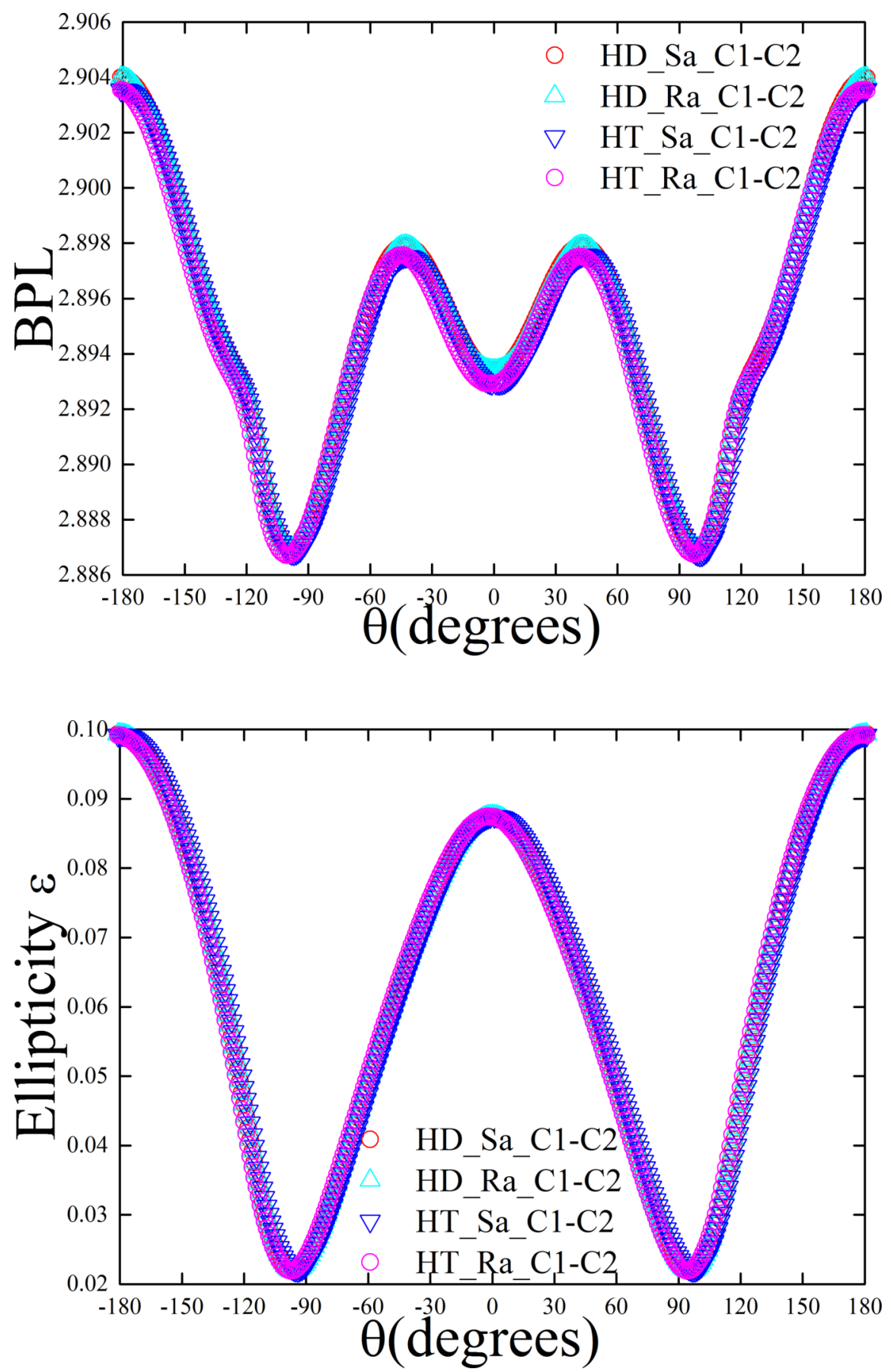

(b) 

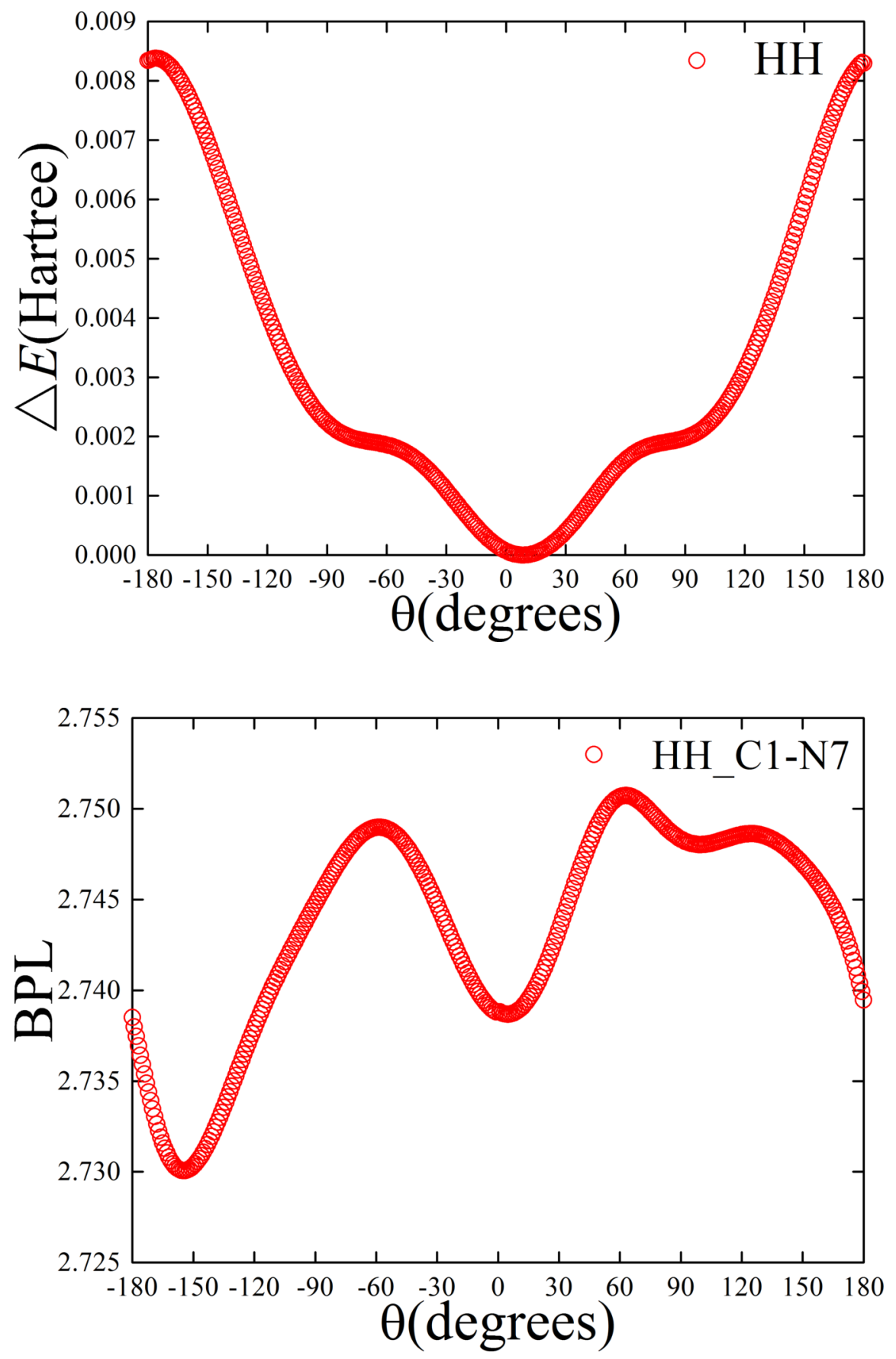


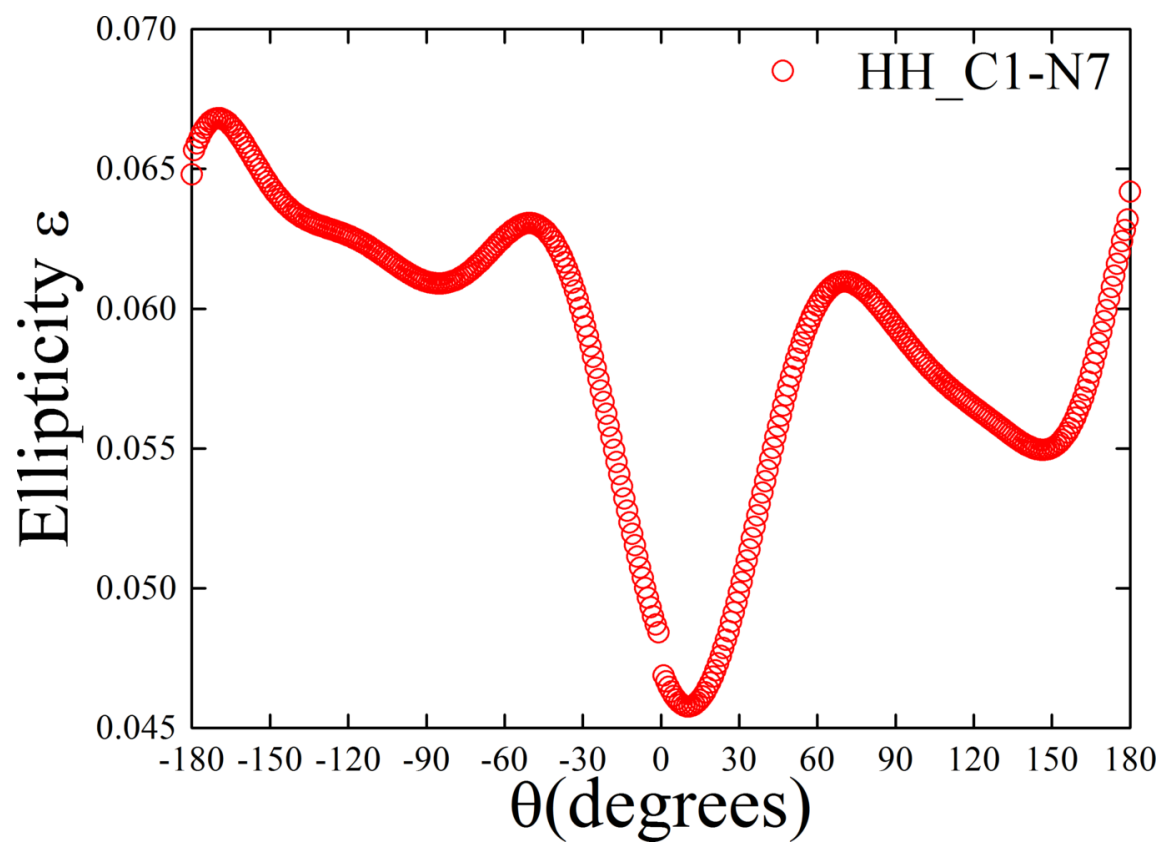

(c)

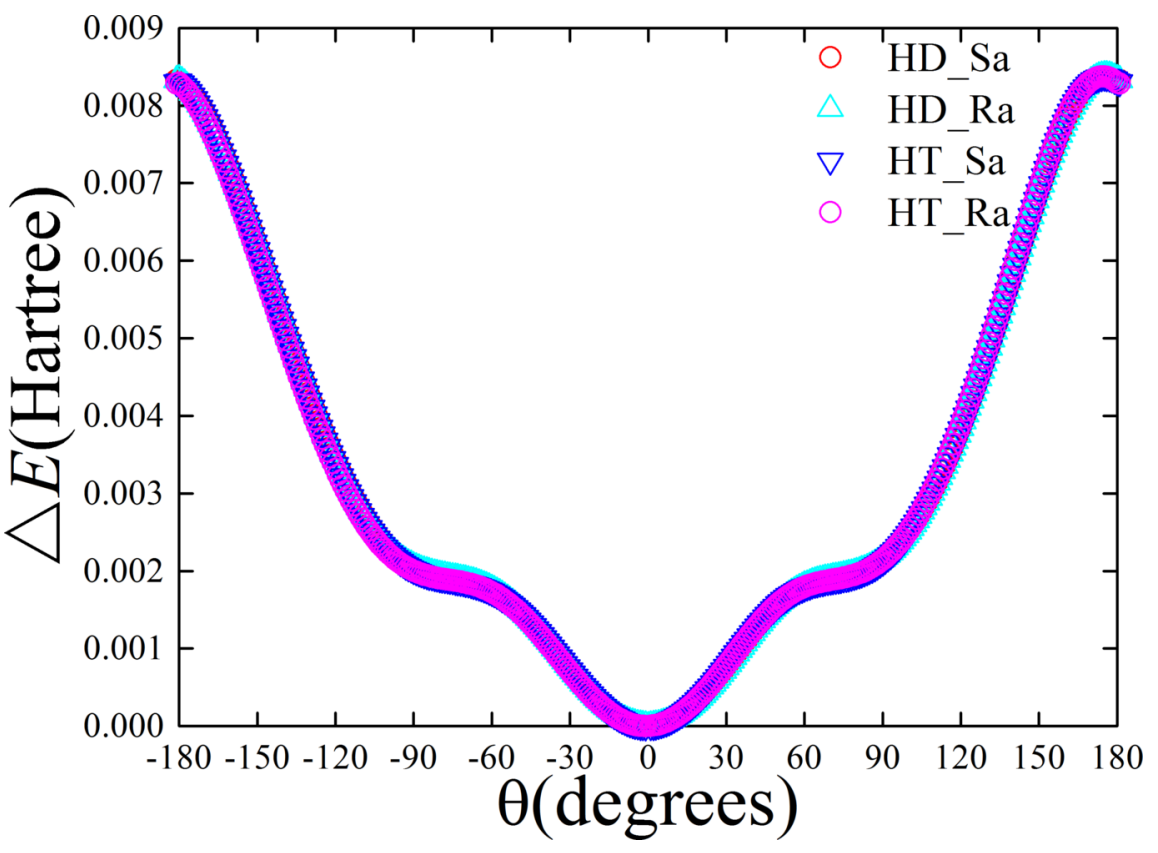



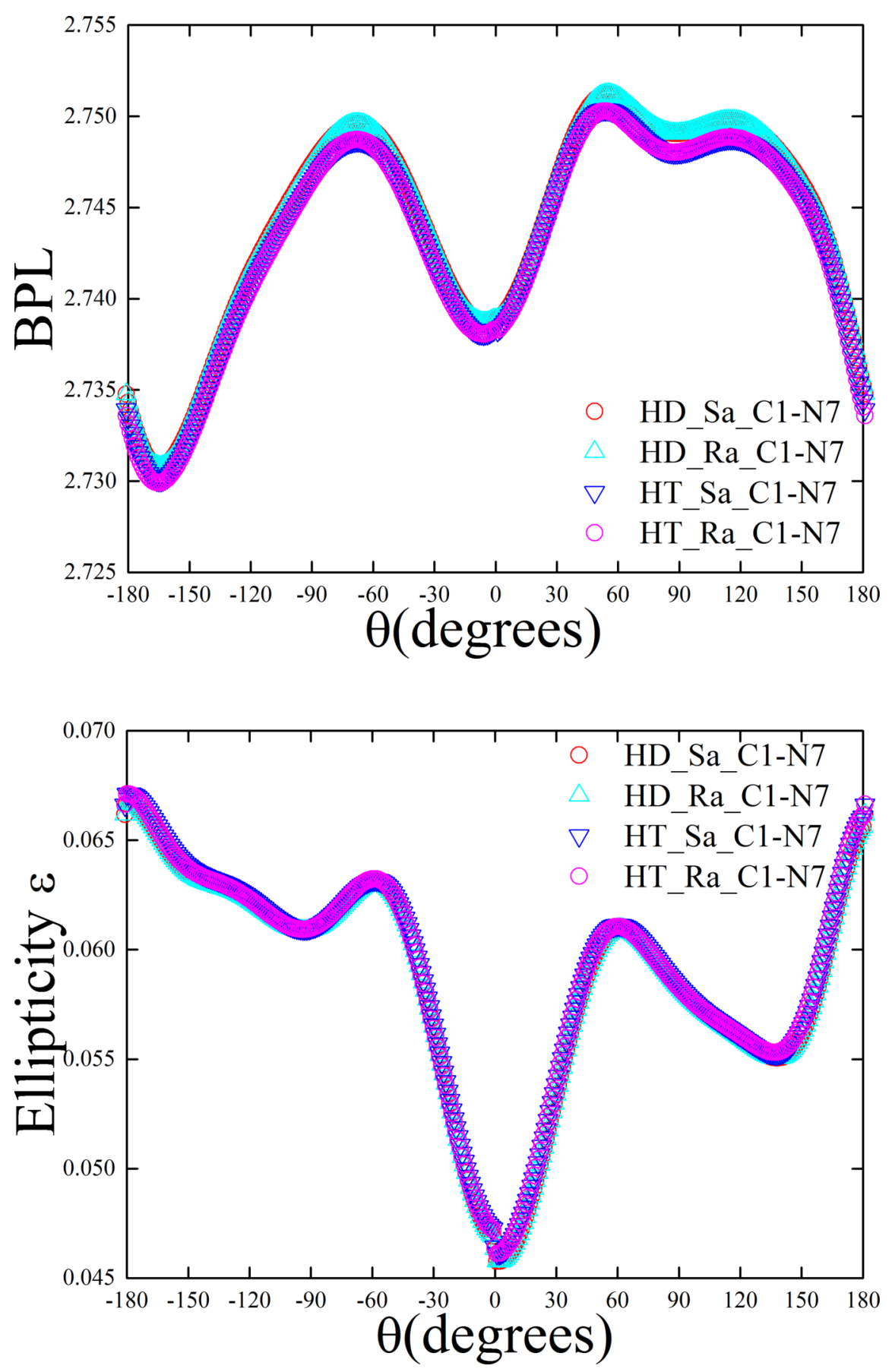

(d)

Figure 1. The variation of the relative energy [?] $E$ of the torsional C1-C2 $B C P$ with the CW $\left(-180.0^{\circ}\right.$ [?] $\vartheta$ [?] $\left.0.0^{\circ}\right)$ and $\mathrm{CCW}\left(0.0^{\mathrm{O}}\right.$ [?] $\vartheta$ [?] 180.0 $\left.{ }^{\mathrm{O}}\right)$ directions of torsion for (left panel), the corresponding variations of the bond-path lengths (BPL) C1-C2 $B C P$ (middle panel) and the ellipticity $\varepsilon$ (right panel) of glycine is presented in Figure 1(a). The plots for the HD and HT isotopomers and $S_{a}$ and $R_{a}$ stereoisomers for the $\mathrm{CW}$ and $\mathrm{CCW}$ directions of the torsional C1-C2 BCP of the variation of the relative energy [?] $E$ (left panel), the variations of the BPL (middle panel) and ellipticity $\varepsilon$ (right panel) are presented in Figure 1(b), also 
see the Scheme 1 for further details. The corresponding plots for the torsional C1-N7 BCP are presented in Figure 1(c) and Figure 1(d) respectively.

Differences in the distance measurements of the minimum energy geometries only become apparent for the introduction of the deuterium and tritium isotopes, see Table $\mathbf{1}(\mathbf{a}-\mathbf{b})$.

Table 1(a) . The distance measures of the isotopomers (HH) of glycine of the structures corresponding to the minimum energy. The values of the inter-nuclear separations that are referred to as the geometric bond-lengths (GBL) bond-path lengths (BPL) (in a.u.) and the partial bond-lengths $(\mathrm{C} 1 / \mathrm{C} 2-B C P, B C P$ $-\mathrm{H} 3)$. Note the BPL $<$ GBL due to the positions of the non-nuclear attractor (NNA) and geometric centre not coinciding for the hydrogen atom.

\begin{tabular}{|c|c|c|c|c|c|}
\hline Isotopomer & $G B L$ & $B P L$ & $(\mathrm{C} 1-B C P, B C P-\mathrm{H} 3 / \mathrm{H} 10)$ & $(\mathrm{C} 1-B C P, B C P-\mathrm{C} 2)$ & $(\mathrm{C} 1-B C P, B C P-\mathrm{N} 7)$ \\
\hline \multicolumn{6}{|l|}{$\overline{H H}$} \\
\hline $\mathrm{C} 1-\mathrm{C} 2$ & 2.8934 & 2.8927 & $(-,-)$ & $(1.3929,1.4999)$ & $(-,-)$ \\
\hline $\mathrm{C} 1-\mathrm{N} 7$ & 2.7389 & 2.7375 & $(-,-)$ & $(-,-)$ & $(1.1980,1.5402)$ \\
\hline C1-H3 & 2.0678 & 2.0360 & $(1.3019,0.7659)$ & $(-,-)$ & $(-,-)$ \\
\hline C1-H10 & 2.0678 & 2.0360 & $(1.3019,0.7659)$ & $(-,-)$ & $(-,-)$ \\
\hline
\end{tabular}

Table 1(b). The distance measures of the $\mathrm{S}_{\mathrm{a}}$ and $\mathrm{R}_{\mathrm{a}}$ for the $H D$ and $H T$ isotopomers of glycine for values of the torsion $\vartheta=0.0^{\circ}$, see the caption of Table $\mathbf{1}(\mathbf{a})$ for further details.

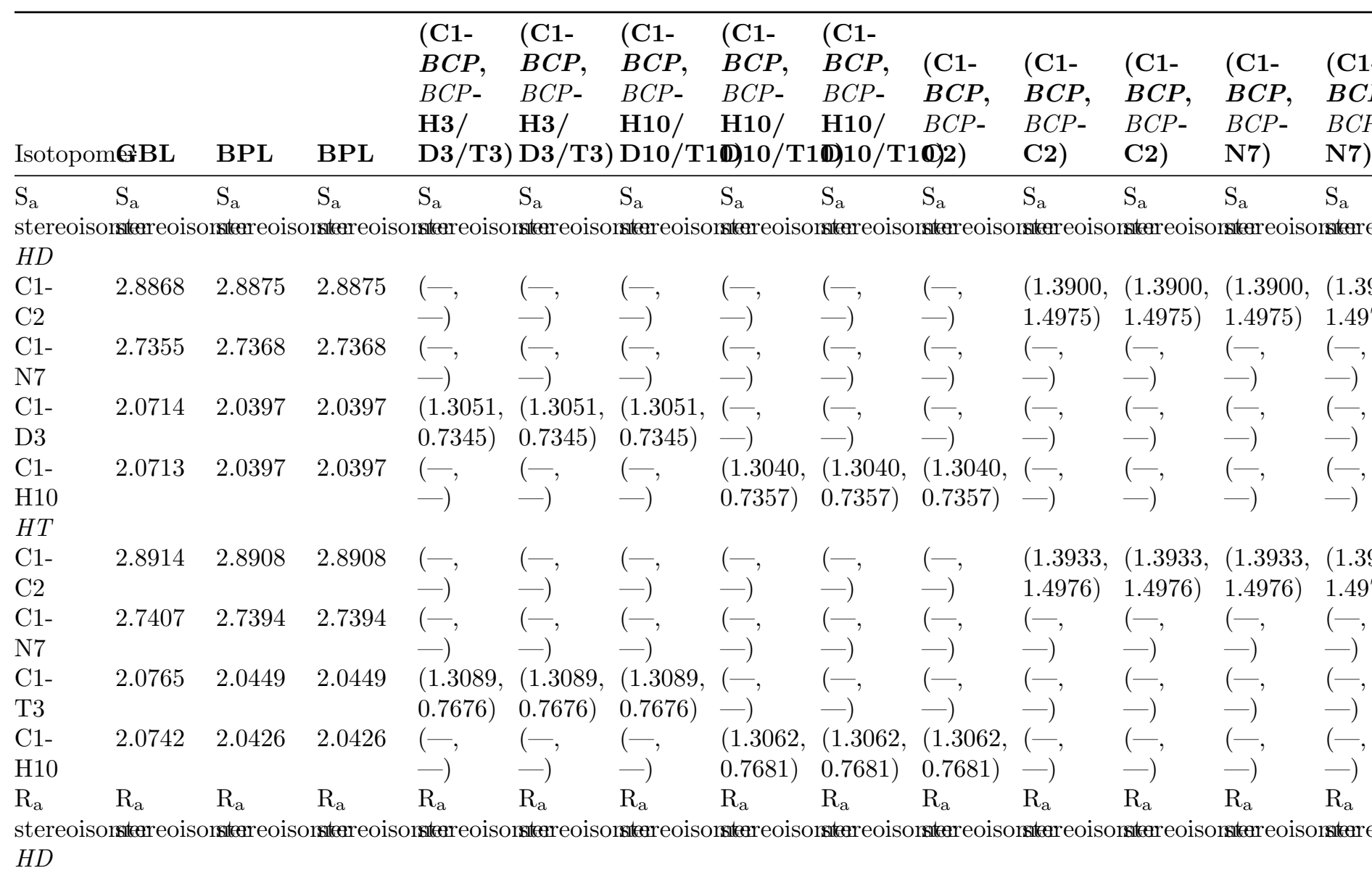




\begin{tabular}{|c|c|c|c|c|c|c|c|c|c|c|c|c|c|}
\hline Isoto & BL & BPL & BPL & $\begin{array}{l}\text { (C1- } \\
\text { BCP, } \\
B C P- \\
\text { H3/ } \\
\text { D3/T3) }\end{array}$ & $\begin{array}{l}\text { (C1- } \\
\text { BCP, } \\
B C P- \\
\text { H3/ } \\
\text { D3/T3) }\end{array}$ & $\begin{array}{l}\text { (C1- } \\
\text { BCP, } \\
B C P- \\
\text { H10/ } \\
\text { D10/T1 }\end{array}$ & $\begin{array}{l}\text { (C1- } \\
B C P, \\
B C P- \\
\text { H10/ } \\
\mathbf{1 D} 10 / \mathrm{T} 1\end{array}$ & $\begin{array}{l}\text { (C1- } \\
\text { BCP, } \\
B C P- \\
\text { H10/ } \\
\text { 1D)10/T1 }\end{array}$ & $\begin{array}{c}(\mathrm{C} 1- \\
B C P, \\
B C P- \\
\text { 10)2 })\end{array}$ & $\begin{array}{l}(\mathbf{C} 1- \\
\mathbf{B C P}, \\
B C P- \\
\text { C2) }\end{array}$ & $\begin{array}{l}(\mathbf{C} 1- \\
\mathbf{B C P}, \\
B C P- \\
\mathbf{C 2})\end{array}$ & $\begin{array}{l}\text { (C1- } \\
\mathbf{B C P}, \\
B C P- \\
\text { N7) }\end{array}$ & $\begin{array}{l}B C \\
B C \\
\text { N7 }\end{array}$ \\
\hline $\begin{array}{l}\text { C1- } \\
\text { C2 }\end{array}$ & 2.8908 & 2.8914 & 2.8914 & $(-$, & $($ - & $($ - & $($ - & $($ - & $(-$, & $\begin{array}{l}(1.3935, \\
1.4980)\end{array}$ & $\begin{array}{l}(1.3935 \\
1.4980)\end{array}$ & $\begin{array}{l}(1.3935 \\
1.4980)\end{array}$ & \\
\hline $\begin{array}{l}\text { C1- } \\
\text { N7 }\end{array}$ & 2.7394 & 2.7407 & 2.7407 & $($ - & $(-$, & $($ - & $($ - & $($ - & $($ - & $($ 一) & $(-)$ & $(-$, & \\
\hline $\begin{array}{l}\text { C1- } \\
\text { H3 }\end{array}$ & 2.0742 & 2.0426 & 2.0426 & $\begin{array}{l}(1.3063 \\
0.7363)\end{array}$ & $\begin{array}{l}(1.3063 \\
0.7363)\end{array}$ & $\begin{array}{l}(1.3063 \\
0.7363)\end{array}$ & $\stackrel{(-}{-}$ & $\stackrel{(-}{-}$ & $($ - & $(-)$ & $(-)$ & $(-$, & \\
\hline $\begin{array}{l}\text { C1- } \\
\text { D10 }\end{array}$ & 2.0765 & 0449 & .0449 & $(-)$ & $(-)$ & $(-)$ & $\begin{array}{l}(1.3091 \\
0.7359)\end{array}$ & $\begin{array}{l}(1.3091 \\
0.7359)\end{array}$ & $\begin{array}{l}\text { (1.3091, } \\
0.7359)\end{array}$ & $(-$, & $(-$, & $(-$, & \\
\hline C1- & 2.8914 & 8908 & 8908 & $($ - & $($ - & $($ - & $($ - & $($ - & $($ - & $\begin{array}{l}\text { (1.3933, } \\
1.4976)\end{array}$ & $\begin{array}{l}(1.3933 \\
1.4976)\end{array}$ & $\begin{array}{l}(1.3933 \\
1.4976)\end{array}$ & $\begin{array}{l}(1 \\
1 .\end{array}$ \\
\hline C1- & 2.7407 & 2.7394 & 2.7394 & $(-$, & $(-$, & $($ - & $($ - & (一) & (一) & $($ - & $(-)$ & $(-$, & \\
\hline C1- & 2.0742 & 2.0426 & 2.0426 & $\begin{array}{l}(1.3062, \\
0.7681)\end{array}$ & $\begin{array}{l}(1.3062 \\
0.7681)\end{array}$ & $\begin{array}{l}(1.3062 \\
0.7681)\end{array}$ & $(-)$ & $(-$, & $($ - & $($ - & $(-$, & $(-)$ & (一) \\
\hline $\begin{array}{l}\text { C1- } \\
\text { T10 }\end{array}$ & 2.0765 & 2.0449 & 2.0449 & $(-$, & $(-)$ & $(-$, & $\begin{array}{l}(1.3089 \\
0.7676)\end{array}$ & $\begin{array}{l}(1.3089 \\
0.7676)\end{array}$ & $\begin{array}{l}(1.3089 \\
0.7676)\end{array}$ & $(-$, & $(-$, & $($ - & $(-)$ \\
\hline
\end{tabular}

\subsection{The stress tensor trajectories for the isotopomers of formally achiral molecules}

The analysis for the dominant, i.e. the strongest torsional bond the C1-N7 BCP used to construct the chirality $\mathrm{C}_{\sigma}$, bond-twist $\mathrm{T}_{\sigma}$, bond-flexing $\mathrm{F}_{\sigma}$ and bond-axiality $\mathrm{A}_{\sigma}$ is presented in Figures 2-3and Table $\mathbf{2}(\mathbf{a}-\mathbf{b})$ and Table $\mathbf{3}(\mathbf{a}-\mathbf{b})$. The geometries of the torsional scans are provided in the Supplementary Materials S2.

For the torsional C1-C2 $B C P$ the presence of non-overlapping $\mathrm{T}_{\sigma}(\mathrm{s})$ for the $\mathrm{CCW}$ and $\mathrm{CW}$ torsions demonstrates the uniqueness of the $\mathrm{T}_{\sigma}(\mathrm{s})$ given the highly symmetrical positioning of the $\mathrm{C} 1-\mathrm{C} 2 \mathrm{BCP}$ along the containing bond-path, seeScheme 1 and Figures S3 of the Supplementary Materials S3 . The stress tensor trajectories $\mathrm{T}_{\sigma}(\mathrm{s})$ of the torsional $\mathrm{C} 1-\mathrm{C} 2 \mathrm{BCP}$ for glycine without isotopic substitution $(\mathrm{HH})$ displays a much smaller extent than occurs with the substitution of the deuterium (HD) or tritium (HT) isotopes. In all three cases $\mathrm{HH}, \mathrm{HD}$ and $\mathrm{HT}$ we can distinguish the presence of the $\mathrm{CCW}$ and $\mathrm{CW}$ directions of torsions by examination of the $\mathrm{C} 1-\mathrm{C} 2 B C P \mathrm{~T}_{\sigma}(\mathrm{s})$. The $\mathrm{S}_{\mathrm{a}}$ and $\mathrm{R}_{\mathrm{a}}$ stress tensor trajectories $\mathrm{T}_{\sigma}(\mathrm{s})$ of the torsional C1-C2 BCP , appear to be indistinguishable for the HD glycine, but not for the HT glycine. The $\mathrm{T}_{\sigma}(\mathrm{s})$ of the non-torsional C-H3/10/D3/T3 BCPsoccur in response to the torsional C1-C2 BCP , see Figure S4 of the Supplementary Materials S4 . The $\mathrm{T}_{\sigma}(\mathrm{s})$ of the C-D3/T3 $B C P$ corresponding to the DH and DT are larger in extent for the $\mathrm{HH}$ glycine. The chirality $\mathrm{C}_{\sigma}$ determined by the torsional $\mathrm{C} 1-\mathrm{C} 2 \mathrm{BCP}$ for the formally achiral HH glycine (0.057) in Table S3 of theSupplementary Materials S3 is comparable to the torsional C1-C2BCP in lactic acid that can be seen from inspection of Table $\mathbf{1}$ in some of the current authors previous work ${ }^{46}$ to be 0.078 and -0.077 for the $\mathrm{S}$ and $\mathrm{R}$ stereoisomers respectively. 

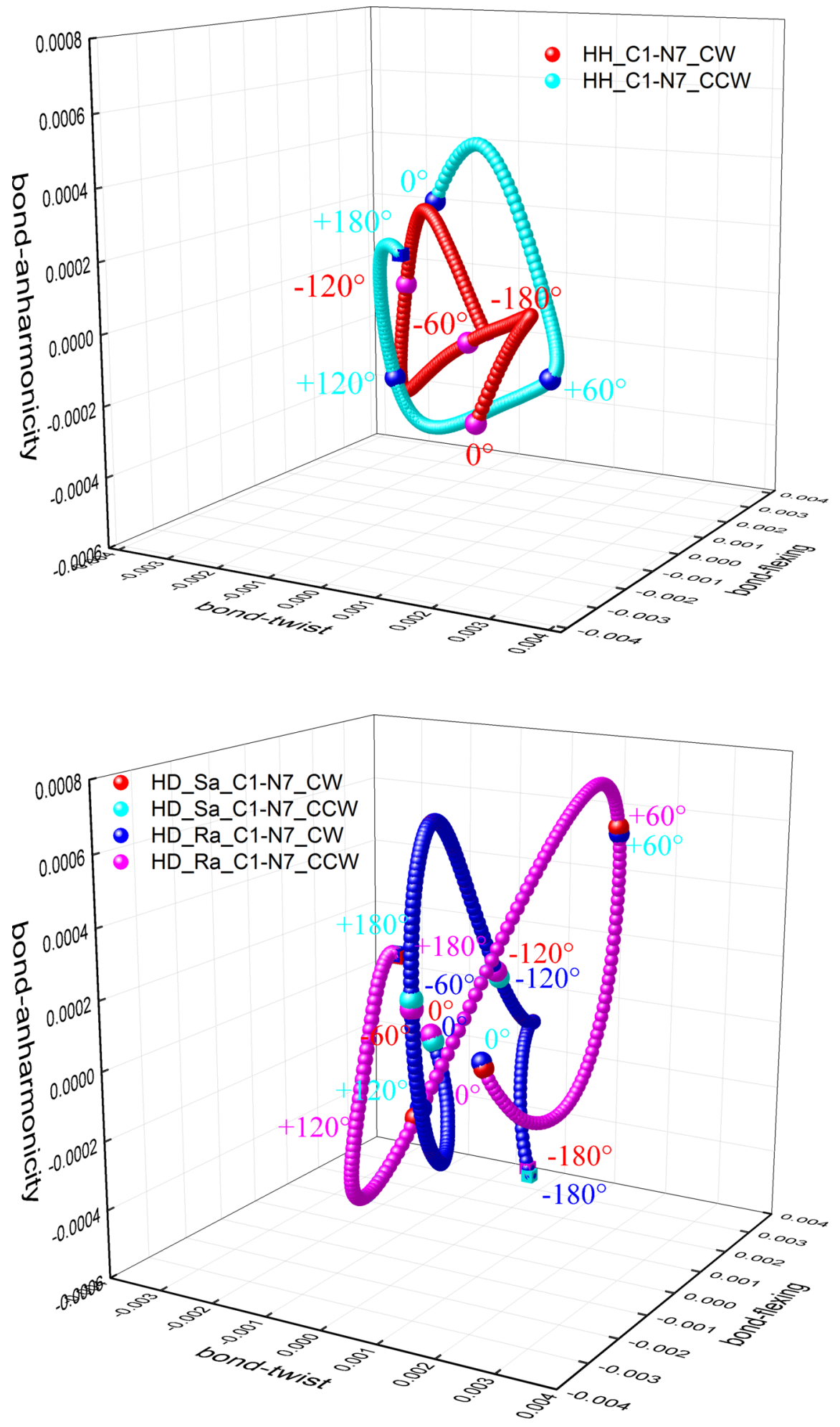


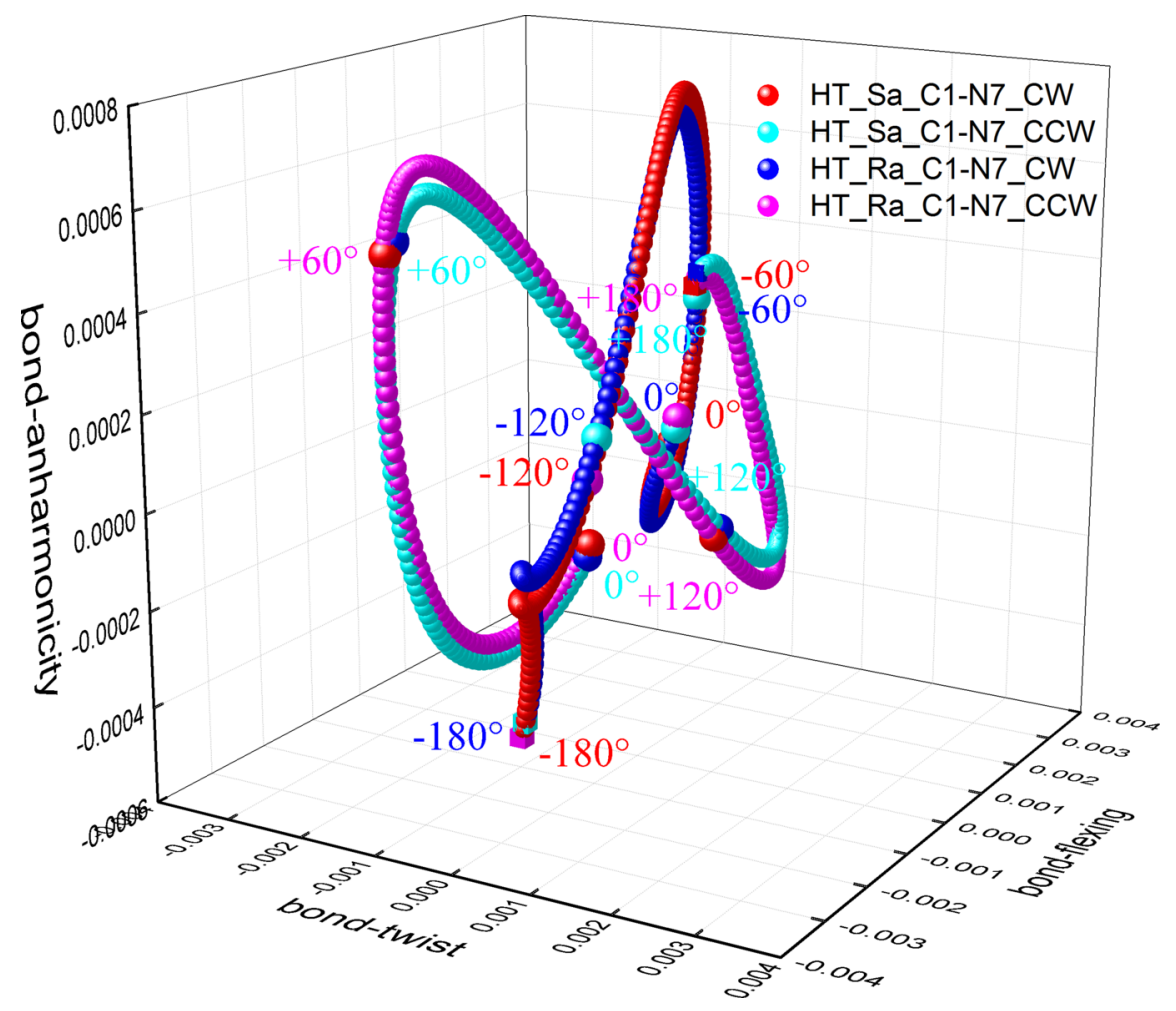

Figure 2 . The stress tensor trajectories $\mathrm{T}_{\sigma}(\mathrm{s})$ of the $\mathrm{CW}$ and $\mathrm{CCW}$ directions of torsion of the torsional C1-N7BCP bond-path for glycine (HH) (left panel) and HD (middle panel), HT(right panel) isotopomers of glycine are presented, see the caption of Figure $\mathbf{1}$ for further details. 

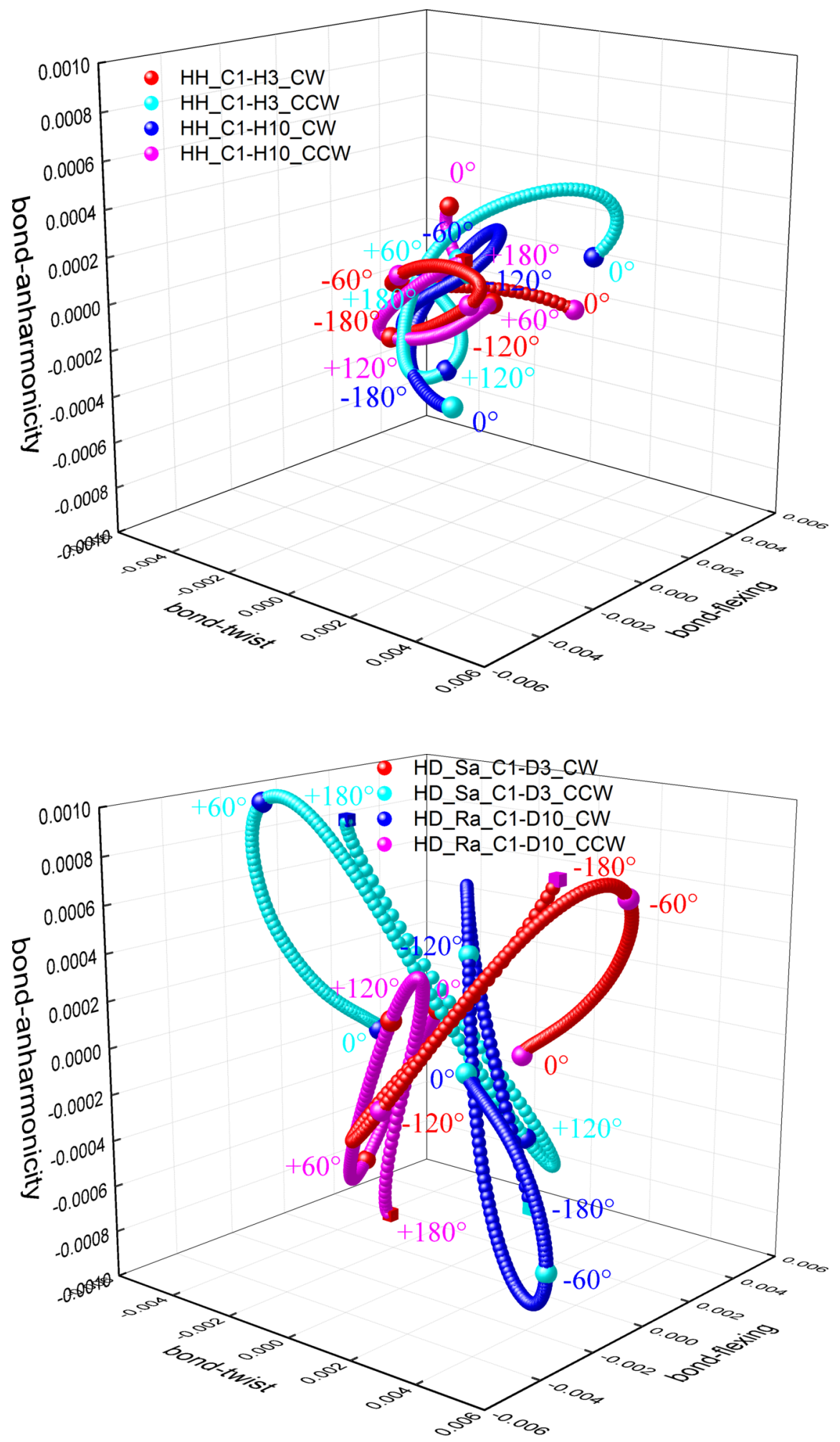


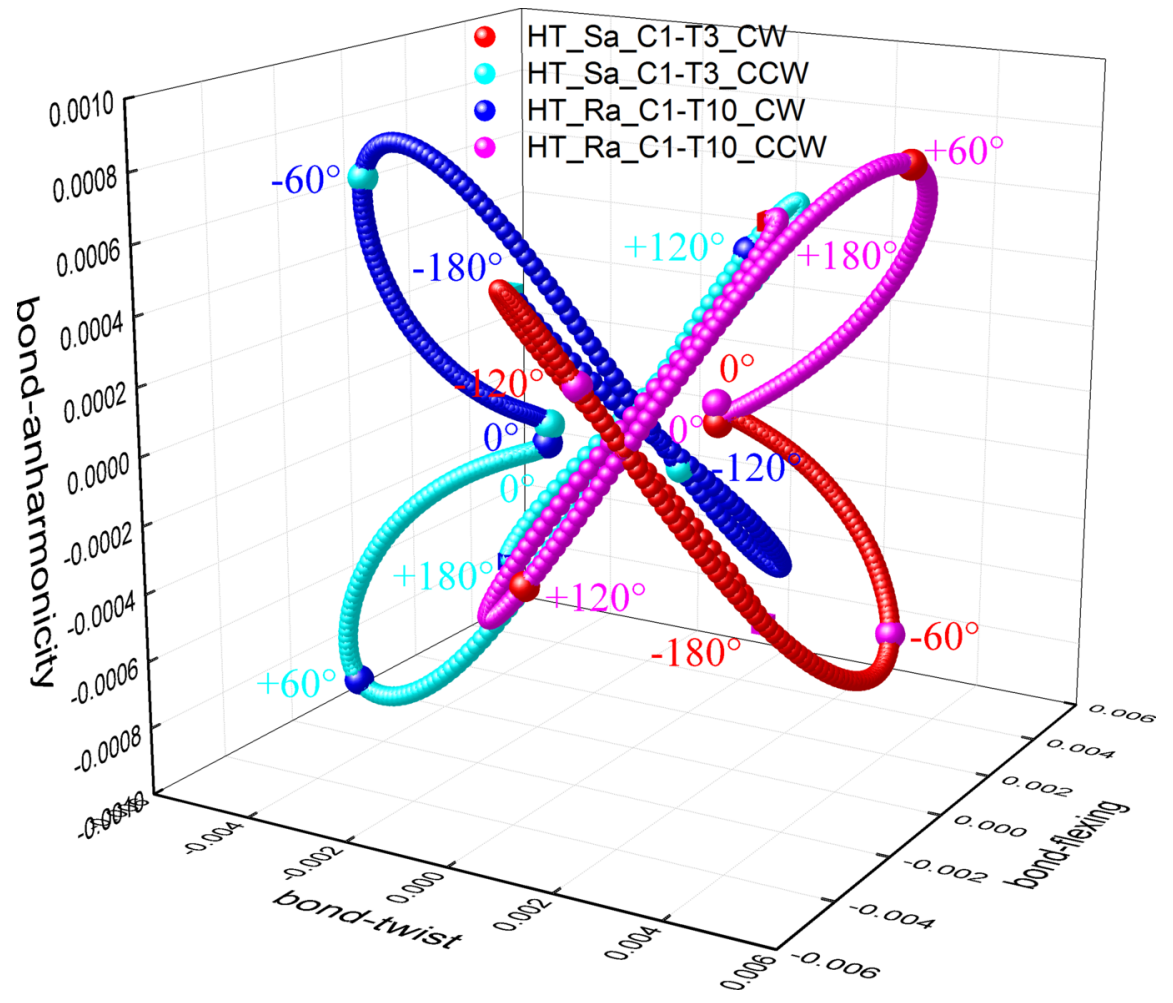

(a)

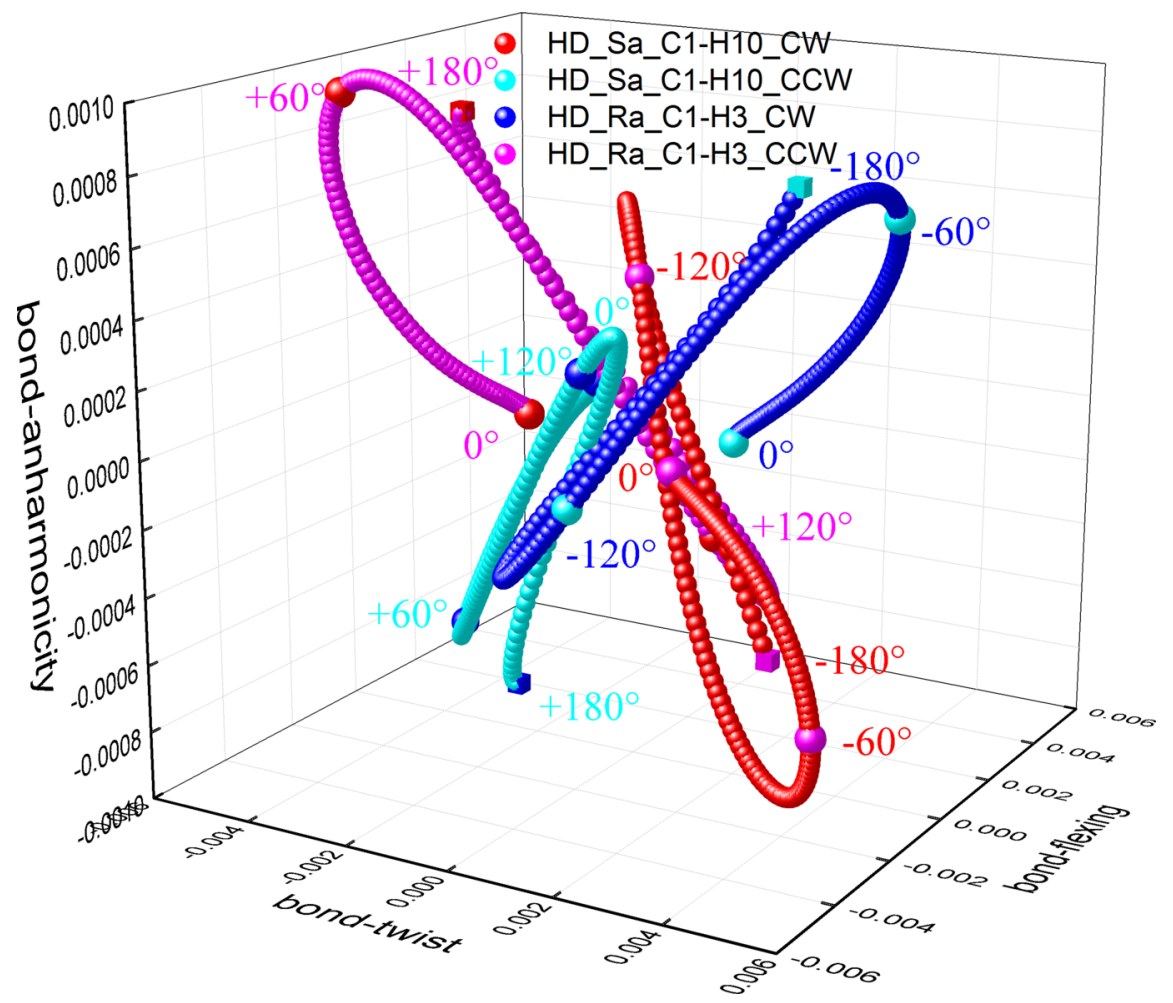




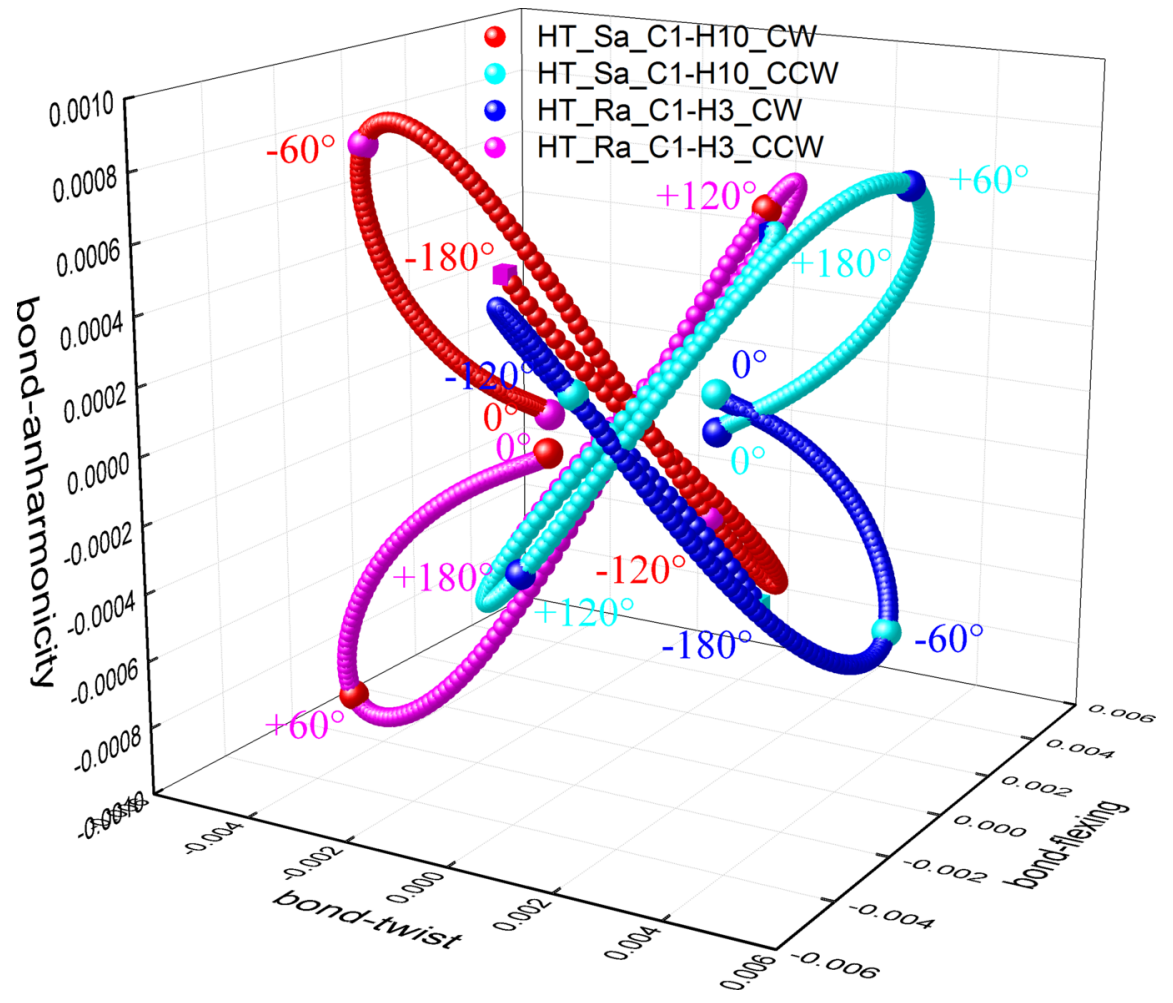

(b)

Figure 3. The stress tensor trajectories $\mathrm{T}_{\sigma}(s)$ of the $\mathrm{CW}\left(-180.0^{\circ}[?] \vartheta[?] \quad 0.0^{\circ}\right)$ and $\mathrm{CCW}\left(0^{\circ}[?] \vartheta[?]\right.$ $180.0^{\circ}$ ) rotations of the $\mathrm{C} 1-\mathrm{H} 3 \mathrm{BCP}$ bond-path and $\mathrm{C} 1-\mathrm{H} 10 B C P$ bond-path of $\mathrm{C} 1-\mathrm{N} 7 \mathrm{BCP}$ torsional bonds for glycine (left panel), the corresponding plots for the C1-D3/D10 $B C P$ (middle panel) and C1-T3/T10 $B C P$ (right panel) are presented in sub-figure (a) . The $\mathrm{T}_{\sigma}(s)$ of the non-isotopomer $\mathrm{C}-\mathrm{H} 3 / 10 B C P \mathrm{~s}$ are presented in sub-figure(b), also see the caption of Figure $\mathbf{1}$ for further details.

Table 2(a). The maximum projections $\mathrm{T}_{\sigma}(s)_{\max }=\left\{\left(\boldsymbol{\varepsilon}_{1 \sigma}[;] \delta \rho\right)_{\mu \alpha \xi}-\beta o v \delta-\tau \omega \iota \sigma \tau,\left(\boldsymbol{\varepsilon}_{2 \sigma}[;] \delta \rho\right)_{\mu \alpha \xi}-\beta o v \delta-\right.$ $\varphi \lambda \varepsilon \xi_{L \nu \gamma},\left(\epsilon_{3 \sigma} . \delta \rho\right)_{\mu \alpha \xi}$-bond-axiality $\}$ values for the C1-N7 BCP, C1-H3/D3/T3 BCP, C1-H10/D10/T10 $B C P, \mathrm{C} 2-\mathrm{O} 5 \mathrm{BCP}$ and $\mathrm{O} 5-\mathrm{H} 6 \mathrm{BCP}$ bond-paths of the $\mathrm{CW}$ and $\mathrm{CCW}$ torsions of the C1-N7 BCP bond-path of the isotopomers of glycine, all entries are multiplied by $10^{3}$.

$\mathrm{T}_{\sigma}(s)_{\max }$

\begin{tabular}{lllll}
\hline $\mathbf{S}_{\mathbf{a}} \mathbf{R}_{\mathbf{a}}$ & $\mathbf{S}_{\mathbf{a}} \mathbf{R}_{\mathbf{a}}$ & $\mathbf{S}_{\mathbf{a}} \mathbf{R}_{\mathbf{a}}$ & $\mathbf{S}_{\mathbf{a}} \mathbf{R}_{\mathbf{a}}$ & $\mathbf{S}_{\mathbf{a}} \mathbf{R}_{\mathbf{a}}$ \\
Isotopomer & $\mathbf{C W}$ & $\mathbf{C W}$ & $\mathbf{C C W}$ & $\mathbf{C C W}$ \\
$H D$ & $H D$ & $H D$ & $H D$ & $H D$ \\
$\mathrm{C} 1-\mathrm{CW}$ & $\{1.25969,6.20430,1.17453\}$ & $\{1.25969,6.20430,1.17453\}$ & $\{1.46775,6.66070,0.98733\}$ & $\{1.46775,6.660$ \\
$\mathrm{C} 1-\mathrm{H} 3$ & $\{-,-,-\}$ & $\{-,-,-\}$ & $\{-,-\}$ & $\{-\}$, \\
$\mathrm{C} 1-\mathrm{D} 3$ & $\{5.36654,4.61234,1.11207\}$ & $\{5.36654,4.61234,1.11207\}$ & $\{6.31495,4.49247,1.58430\}$ & $\{6.31495,4.492$ \\
$\mathrm{C} 1-\mathrm{H} 10$ & $\{5.98267,4.21167,1.47115\}$ & $\{5.98267,4.21167,1.47115\}$ & $\{5.65679,5.06483,1.31864\}$ & $\{5.65679,5.064$ \\
C1-D10 & $\{-,-,-\}$ & $\{-,-,-\}$ & $\{-,-,-\}$ & $\{-,-\}$, \\
C2-O5 & $\{2.82360,3.94348,2.87514\}$ & $\{2.82360,3.94348,2.87514\}$ & $\{3.32765,4.37802,3.93280\}$ & $\{3.32765,4.378$ \\
O5-H6 & $\{25.61721,1.11027,6.38973\}$ & $\{25.61721,1.11027,6.38973\}$ & $\{28.60879,1.67954,8.25715\}$ & $\{28.60879,1.67$ \\
$H T$ & $H T$ & $H T$ & $H T$ & $H T$ \\
C1-N7 & $\{1.34308,6.22003,1.20499\}$ & $\{1.34308,6.22003,1.20499\}$ & $\{1.31574,6.81512,0.97125\}$ & $\{1.31574,6.815$ \\
C1-H3 & $\{-,-,-\}$ & $\{-,-,-\}$ & $\{-,-,-\}$ & $\{-,-,-\}$
\end{tabular}




$\begin{array}{ll}\text { C1-T3 } & \{5.34733,4.68833,1.17787\} \\ \text { C1-H10 } & \{5.86356,4.46846,1.42940\} \\ \text { C1-T10 } & \{-,-,-\} \\ \text { C2-O5 } & \{2.12201,4.47510,2.14513\} \\ \text { O5-H6 } & \{25.42079,0.86864,4.96079\} \\ \text { HH } & H H \\ \text { C1-N7 } & \{3.26019,1.60696,0.54036\} \\ \text { C1-H3 } & \{3.33726,3.75752,0.35676\} \\ \text { C1-H10 } & \{3.90399,4.38773,0.55697\} \\ \text { C2-O5 } & \{2.82335,3.94348,2.87501\} \\ \text { O5-H6 } & \{25.61750,1.10918,6.38972\}\end{array}$

$\{5.34733,4.68833,1.17787\}$

$\{5.86356,4.46846,1.42940\}$

$\{-,-,-\}$

$\{2.12201,4.47510,2.14513\}$

$\{25.42079,0.86864,4.96079\}$

CW

$\{3.26019,1.60696,0.54036\}$

$\{3.33726,3.75752,0.35676\}$

$\{3.90399,4.38773,0.55697\}$

$\{2.82335,3.94348,2.87501\}$

$\{25.61750,1.10918,6.38972\}$
$\{6.47606,4.81406,1.47222\}$

$\{5.87796,5.10046,1.19824\}$

$\{-,-,-\}$

$\{2.48854,4.85582,2.75258\}$

$\{27.74975,1.22755,6.00838\}$

CW

$\{3.26019,1.60696,0.54036\}$

$\{3.33726,3.75752,0.35676\}$

$\{3.90399,4.38773,0.55697\}$

$\{2.82335,3.94348,2.87501\}$

$\{25.61750,1.10918,6.38972\}$
$\{6.47606,4.814$

$\{5.87796,5.100$

$\{-,-,-\}$

$\{2.48854,4.855$

$\{27.74975,1.22$ CW

$\{3.44982,1.276$

$\{3.79503,4.681$

$\{4.47024,4.338$

$\{3.32762,4.378$

$\{28.60857,1.67$

Table 2(b). The values of the chirality $\mathrm{C}_{\sigma}=\left[\left(\varepsilon_{1 \sigma}[;] \delta \rho\right)_{\mu \alpha \xi}\right]_{\mathbf{C C W}^{-}}\left[\left(\varepsilon_{1 \sigma}[;] \delta \rho\right)_{\mu \alpha \xi}\right]_{\mathbf{C W}}$, bondflexing $\mathrm{F}_{\sigma}=\left[\left(\varepsilon_{2 \sigma}[;] \delta \rho\right)_{\mu \alpha \xi}\right]_{\mathrm{CCW}^{-}}\left[\left(\varepsilon_{2 \sigma}[;] \delta \rho\right)_{\mu \alpha \xi}\right]_{\mathrm{CW}}$ and bond-axiality $A_{\sigma}=\left[\left(\varepsilon_{3 \sigma}[;] \delta \rho\right)_{\mu \alpha \xi}\right]_{\mathrm{CCW}^{-}}$ $\left[\left(\varepsilon_{3 \sigma}[;] \delta \rho\right)_{\mu \alpha \xi}\right]_{\text {CW }}$ of the torsional C1-N7 BCP for the isotopomers of glycine are presented, all entries are multiplied by $10^{3}$. The stereoisomeric excess $\mathrm{X}_{\sigma}$ is defined as the ratio of the magnitude of the $\mathrm{C}_{\sigma}$ values of the $\mathrm{S}_{\mathrm{a}}$ and $\mathrm{R}_{\mathrm{a}}$ stereoisomers of the torsional C1-N7 BCP.

$\left\{\mathrm{C}_{\sigma}, \mathrm{F}_{\sigma}, \mathrm{A}_{\sigma}\right\}$

\begin{tabular}{llll}
\hline Isotopomer & $\mathbf{S}_{\mathbf{a}}$ & $\mathbf{R}_{\mathbf{a}}$ & $\mathbf{R}_{\mathbf{a}}$ \\
$H D$ & $\{0.20806[\mathbf{S}], 0.45640[\mathbf{S}],-0.18720[\mathbf{R}]\}$ & $\{0.20806[\mathbf{S}], 0.45640[\mathbf{S}],-0.18720[\mathbf{R}]\}$ & $\{0.20819[\mathbf{S}], 0.45631[\mathbf{S}],-0$ \\
$H T$ & $\{-0.02734[\mathbf{R}], 0.59509[\mathbf{S}],-0.23374[\mathbf{R}]\}$ & $\{-0.02734[\mathbf{R}], 0.59509[\mathbf{S}],-0.23374[\mathbf{R}]\}$ & $\{-0.02734[\mathbf{R}], 0.59509[\mathbf{S}],-$ \\
& $\left\{\mathrm{C}_{\sigma}, \mathrm{F}_{\sigma}, \mathrm{A}_{\sigma}\right\}$ & $\left\{\mathrm{C}_{\sigma}, \mathrm{F}_{\sigma}, \mathrm{A}_{\sigma}\right\}$ & $\left\{\mathrm{C}_{\sigma}, \mathrm{F}_{\sigma}, \mathrm{A}_{\sigma}\right\}$ \\
$H H$ & $\{0.18963[\mathbf{S}],-0.33054[\mathbf{R}], 0.17961[\mathbf{S}]\}$ & $\{0.18963[\mathbf{S}],-0.33054[\mathbf{R}], 0.17961[\mathbf{S}]\}$ & $\{0.18963[\mathbf{S}],-0.33054[\mathbf{R}], 0$ \\
\hline
\end{tabular}

We now consider the chirality $\mathrm{C}_{\sigma}$, bond-flexing $\mathrm{F}_{\sigma}$ and bond-axiality $\mathrm{A}_{\sigma}$ values of the dominant torsional C1-N7 $B C P$, see Figure $\mathbf{2}$ andTable 2(b) . The formally achiral HH glycine comprises a significant degree of chirality $\mathrm{C}_{\sigma}(=0.18963)$ for the torsional C1-N7 BCP with $\boldsymbol{\Sigma}_{\boldsymbol{\sigma}}$ character, see Table $\mathbf{2}(\mathbf{a}-\mathbf{b})$. The magnitude of $\mathrm{C}_{\sigma}$ increases similarly with the addition of the $\mathrm{D}$ isotope for both the $\mathrm{S}_{\mathrm{a}}$ and $\mathrm{R}_{\mathrm{a}}$ stereoisomers, where the $\mathrm{R}_{\mathrm{a}}$ stereoisomer possesses the larger value, both stereoisomers are $\boldsymbol{\Sigma}_{\boldsymbol{\sigma}}$ character. The bond-flexing $\mathrm{F}_{\sigma}$ is more affected by the substitution of the $\mathrm{D}$ isotope to $\mathrm{HH}$ glycine than either of the chirality $\mathrm{C}_{\sigma}$ or $\mathrm{A}_{\sigma}$ of the torsional C1-N7BCP , see Table 2(b) .

The substitution of the $\mathrm{T}$ isotope onto the alpha carbon of $\mathrm{HH}$ glycine to replace an $\mathrm{H}$ significantly changes $\mathrm{C}_{\sigma}, \mathrm{F}_{\sigma}$ and $\mathrm{A}_{\sigma}$ values. The $\mathrm{T}$ isotope changes the character of the chirality $\mathrm{C}_{\sigma}$ to $\mathbf{P}_{\sigma}$ character for both the $S_{a}$ and $R_{a}$ stereoisomers and there is a pronounced difference in the magnitude of $C_{\sigma}$, that is significantly lower for the $S_{a}$ stereoisomer. The bond-flexing $F_{\sigma}$ increases in magnitude from $\mathrm{HH}$ glycine through to HD glycine and HT glycine, again the HD glycine values are similar for the $\mathrm{S}_{\mathrm{a}}$ and $\mathrm{R}_{\mathrm{a}}$ stereoisomers with a slight preference for the $\mathrm{S}_{\mathrm{a}}$ stereoisomer. In addition, the bond-flexing $\mathrm{F}_{\sigma}$ changes from $\boldsymbol{\Sigma}_{\boldsymbol{\sigma}}$ character, for $\mathrm{HH}$ glycine, to $\mathbf{P}_{\boldsymbol{\sigma}}$ character for both the $\mathrm{S}_{\mathrm{a}}$ and $\mathrm{R}_{\mathrm{a}}$ stereoisomers of $\mathrm{HD}$ glycine and HT glycine. The bond-axiality $\mathrm{A}_{\sigma}$ increases in magnitude from $\mathrm{HH}$ glycine through to $\mathrm{HD}$ glycine and $\mathrm{S}_{\mathrm{a}}$ stereoisomer of $\mathrm{HT}$ glycine, again the HD glycine values are similar for the $S_{a}$ and $R_{a}$ stereoisomers with a slight preference for the $\mathrm{R}_{\mathrm{a}}$ stereoisomer. The $\mathrm{A}_{\sigma}$ value of the $\mathrm{R}_{\mathrm{a}}$ stereoisomer of $\mathrm{HT}$ glycine decreases significantly below that of $\mathrm{HH}$ glycine. The $\mathrm{A}_{\sigma}$ value of $\mathrm{HH}$ glycine possesses $\boldsymbol{\Sigma}_{\boldsymbol{\sigma}}$ character that changes to $\mathbf{P}_{\boldsymbol{\sigma}}$ character for both the $\mathrm{S}_{\mathrm{a}}$ and $\mathrm{R}_{\mathrm{a}}$ stereoisomers of HD glycine and HT glycine. The stereoisomeric excess $\mathrm{X}_{\sigma}$ values indicate a very slight preference for the $\mathrm{R}_{\mathrm{a}}$ stereoisomer that possesses chirality $\mathrm{C}_{\sigma}$ with $\boldsymbol{\Sigma}_{\boldsymbol{\sigma}}$ character for HD glycine and a very strong preference for the $\mathrm{R}_{\mathrm{a}}$ stereoisomer that possesses chirality $\mathrm{C}_{\sigma}$ with $\mathbf{P}_{\sigma}$ character for HD glycine.

Table 2(c). Values of the bond-twist $T_{\sigma}=\left[\left(\varepsilon_{1 \sigma}[;] \delta \rho\right)_{\mu \alpha \xi}\right]_{\mathbf{C C W}^{-}}\left[\left(\varepsilon_{1 \sigma}[;] \delta \rho\right)_{\mu \alpha \xi}\right]_{\mathbf{C W}}$, bond-flexing $\mathrm{F}_{\sigma}$ and the bond-axiality $\mathrm{A}_{\sigma}$ of the $\mathrm{C}-\mathrm{H} / \mathrm{D} / \mathrm{T} B C P$ bond-paths corresponding to the torsional C1-N7 $B C P$ 
bond-path of the isotopomers of glycine are presented, see the caption of Table 2(b) for further details.

\begin{tabular}{lll}
\hline Isotopomer & $\left\{\mathrm{T}_{\sigma}, \mathrm{F}_{\sigma}, \mathrm{A}_{\sigma}\right\}$ & $\left\{\mathrm{T}_{\sigma}, \mathrm{F}_{\sigma}, \mathrm{A}_{\sigma}\right\}$ \\
\hline HD & $\mathbf{S}_{\mathbf{a}}$ & $\mathbf{R}_{\mathbf{a}}$ \\
C1-H3 & $\{-,-,-\}$ & $\{0.94835,-0.11973,0.47203\}$ \\
C1-D3 & $\{0.94841,-0.11987,0.47223\}$ & $\{-,-,-\}$ \\
C1-H10 & $\{-0.32583,0.85323,-0.15223\}$ & $\{-,-,-\}$ \\
C1-D10 & $\{-,-,-\}$ & $\{-0.32588,0.85326,-0.15251\}$ \\
HT & & \\
C1-H3 & $\{-,-,-\}$ & $\{-,-,-\}$ \\
C1-T3 & $\{1.12873,0.12573,0.29435\}$ & $\{-,-,-\}$ \\
C1-H10 & $\{0.01440,0.63200,-0.23116\}$ & $\{-,-,-\}$ \\
C1-T10 & $\{-,-,-\}$ & $\{-0.00004,0.58866,-0.01290\}$ \\
HH & $\left\{\mathrm{T}_{\sigma}, \mathrm{F}_{\sigma}, \mathrm{A}_{\sigma}\right\}$ & $\left\{\mathrm{T}_{\sigma}, \mathrm{F}_{\sigma}, \mathrm{A}_{\sigma}\right\}$ \\
C1-H3 & $\{0.45777,0.92442,0.36101\}$ & $\{0.45777,0.92442,0.36101\}$ \\
C1-H10 & $\{0.56625,-0.04926,-0.15746\}$ & $\{0.56625,-0.04926,-0.15746\}$ \\
\hline
\end{tabular}

Table 2(d). Values of the bond-twist $\mathrm{T}_{\sigma}$, bond-flexing $\mathrm{F}_{\sigma}$ and the bond-axiality $\mathrm{A}_{\sigma}$ of the $\mathrm{C} 2-\mathrm{O} 5 \mathrm{BCP}$ and O5-H6 BCP bond-paths corresponding to the torsional C1-N7 BCP bond-path of the isotopomers of glycine are presented, see the caption of Table $\mathbf{2}(\mathbf{b})$ for further details.

\begin{tabular}{llll}
\hline Isotopomer & $\left\{\mathrm{T}_{\sigma}, \mathrm{F}_{\sigma}, \mathrm{A}_{\sigma}\right\}$ & $\left\{\mathrm{T}_{\sigma}, \mathrm{F}_{\sigma}, \mathrm{A}_{\sigma}\right\}$ & $\left\{\mathrm{T}_{\sigma}, \mathrm{F}_{\sigma}, \mathrm{A}_{\sigma}\right\}$ \\
\hline $\mathrm{HD}$ & $\mathbf{S}_{\mathbf{a}}$ & $\mathbf{R}_{\mathbf{a}}$ & $\mathbf{R}_{\mathbf{a}}$ \\
C2-O5 & $\{0.50405,0.43454,1.05766\}$ & $\{0.50426,0.43460,1.05653\}$ & $\{0.50426,0.43460,1.05653\}$ \\
O5-H6 & $\{2.99158,0.56927,1.86742\}$ & $\{2.99145,0.57040,1.86742\}$ & $\{2.99145,0.57040,1.86742\}$ \\
HT & & & \\
C2-O5 & $\{0.36653,0.38072,0.60745\}$ & $\{0.41177,0.24549,0.59381\}$ & $\{0.41177,0.24549,0.59381\}$ \\
O5-H6 & $\{2.32896,0.35891,1.04759\}$ & $\{1.83414,0.38584,1.00560\}$ & $\{1.83414,0.38584,1.00560\}$ \\
HH & $\left\{\mathrm{T}_{\sigma}, \mathrm{F}_{\sigma}, \mathrm{A}_{\sigma}\right\}$ & $\left\{\mathrm{T}_{\sigma}, \mathrm{F}_{\sigma}, \mathrm{A}_{\sigma}\right\}$ & \\
C2-O5 & $\{0.50427,0.43458,1.05765\}$ & $\{0.50427,0.43458,1.05765\}$ & $\{0.50427,0.43458,1.05765\}$ \\
O5-H6 & $\{2.99107,0.57022,1.86759\}$ & $\{2.99107,0.57022,1.86759\}$ & $\{2.99107,0.57022,1.86759\}$ \\
\hline
\end{tabular}

Consideration of the non-torsional $\mathrm{C} 1-\mathrm{H} / \mathrm{D} / \mathrm{T} B C P s$ is presented inFigure 3, Table 2(c) and Table 2(d) . For HD glycine the values of $\mathrm{T}_{\sigma}, \mathrm{F}_{\sigma}$ and $\mathrm{A}_{\sigma}$ are all slightly larger for C1-D3 $B C P$ (the $\mathrm{R}_{\mathrm{a}}$ stereoisomer) than for the corresponding $\mathrm{T}_{\sigma}, \mathrm{F}_{\sigma}$ and $\mathrm{A}_{\sigma}$ values of $\mathrm{C} 1-\mathrm{H} 3 \mathrm{BCP}$ (the $\mathrm{S}_{\mathrm{a}}$ stereoisomer), seeTable 2(c) . The same correspondence is found for the C1-D10BCP. This does not hold for the HT glycine where the opposite effect is found for the C1-T10 $B C P$, where all three of the $\mathrm{T}_{\sigma}, \mathrm{F}_{\sigma}$ and $\mathrm{A}_{\sigma}$ values are smaller for the C1-T10 $B C P$ compared with the C1-H10 $B C P$. A mixed effect is found for the dominance of the $\mathrm{T}_{\sigma}$, $\mathrm{F}_{\sigma}$ and $\mathrm{A}_{\sigma}$ values of the $\mathrm{C} 1-\mathrm{T} 3 \mathrm{BCP}$ compared with the $\mathrm{C} 1-\mathrm{H} 3 \mathrm{BCP}$. Particularly high values of the $\mathrm{F}_{\sigma}$ and $\mathrm{A}_{\sigma}$ are observed for the $\mathrm{O} 5-\mathrm{H} 6 \mathrm{BCP}$ and the $\mathrm{C} 2-\mathrm{O} 5 \mathrm{BCP}$ that reduce significantly with the addition of the T isotope to X3 and X10 sites. Despite the remotely positioned location of the C2-O5 $B C P$ and O5-H6 $B C P$ from the site of the $\mathrm{D}$ and $\mathrm{T}$ substitutions, the effect of the $\mathrm{T}$ isotope in particular is apparent where a significant reduction of the $\mathrm{T}_{\sigma}, \mathrm{F}_{\sigma}$ and $\mathrm{A}_{\sigma}$ values of both the $\mathrm{C} 2-\mathrm{O} 5$ BCP and $\mathrm{O} 5-\mathrm{H} 6$ BCP compared with D and T, see Table 2(d). These observations are relevant for the interpretation of the vibrational mode coupling, which affects both IR and CD spectra. This is because the high values of $\mathrm{F}_{\sigma}$ and $\mathrm{A}_{\sigma}$ of both the $\mathrm{C} 2-\mathrm{O} 5 \mathrm{BCP}$ and $\mathrm{O} 5-\mathrm{H} 6 \mathrm{BCP}$ that are consistent with the $\mathrm{C} 2-\mathrm{O} 5-\mathrm{H} 6$ undergoing a bending vibration which is coupled to the methylene group wagging which is thus expected to be preserved upon deuteration at $\mathrm{C} 1$. Thelowering of the corresponding values of $\mathrm{F}_{\sigma}$ and $\mathrm{A}_{\sigma}$ for the $\mathrm{T}$ isotope indicates that the $\mathrm{C} 2-\mathrm{O} 5-\mathrm{H} 6$ 
bending vibration is uncoupled from that of C1-HT wagging.

\section{Conclusions}

Using Next generation QTAIM we discovered $\boldsymbol{\Sigma}_{\sigma}$ character chirality $\mathrm{C}_{\sigma}$ for the dominant torsional C1$\mathrm{N} 7 B C P$ in $\mathrm{HH}$ (ordinary) i.e. formally achiral glycine. The associated bond-flexing $\mathrm{F}_{\sigma}$ and bond-axiality $\mathrm{A}_{\sigma}$ possessed $\mathbf{P}_{\sigma}$ and $\boldsymbol{\Sigma}_{\boldsymbol{\sigma}}$ character respectively. The introduction of the $\mathrm{D}$ and $\mathrm{T}$ isotopes caused a reversal of the $\mathbf{P}_{\boldsymbol{\sigma}}$ and $\boldsymbol{\Sigma}_{\boldsymbol{\sigma}}$ character torsional C1-N7 BCP of the bond-flexing $\mathrm{F}_{\boldsymbol{\sigma}}$ and bond-axiality $\mathrm{A}_{\sigma}$. The presence of the $\boldsymbol{\Sigma}_{\boldsymbol{\sigma}}$ character chirality $\mathrm{C}_{\sigma}$ found for ordinary $\mathrm{HH}$ glycine was preserved after the addition of the D isotope but after replacement of the $\mathrm{D}$ isotope with the $\mathrm{T}$ isotope the chirality $\mathrm{C}_{\sigma}$ reversed to $\mathbf{P}_{\sigma}$ character. This reversal of the chirality $\mathrm{C}_{\sigma}$, depending on the presence of a $\mathrm{D}$ or $\mathrm{T}$ isotope on the alpha carbon adds to the debate as to the nature of the extraterrestrial origins of chirality in simple amino acids. This is since either $\mathrm{D}$ or $\mathrm{T}$ isotopes of hydrogen may be present, albeit at rather low concentrations, in these hostile extraterrestrial environments.

We also considered the effects of the addition of the $\mathrm{D}$ and $\mathrm{T}$ isotopes on the bond-twist $\mathrm{T}_{\sigma}$, bond-flexing $\mathrm{F}_{\sigma}$ and the bond-axiality $\mathrm{A}_{\sigma}$ of the non-torsional $\mathrm{C}-\mathrm{H} / \mathrm{D} / \mathrm{T} B C P \mathrm{~s}$.

This theoretical analysis represents a new challenge for the eventual observation of glycine chiral properties. Interestingly, for vibrational spectroscopies the "classic" nuclear isotopic effects can be also exploited to identify any new weak signals not corresponding to the energy ranges of the main HH species.

As an example, the effect of the addition of the $\mathrm{T}$ isotope to the $\mathrm{X} 3$ and $\mathrm{X} 10$ sites on lowering the $\mathrm{F}_{\sigma}$ and $\mathrm{A}_{\sigma}$ values for the $\mathrm{O} 5-\mathrm{H} 6 \mathrm{BCP}$ and $\mathrm{C} 2-\mathrm{O} 5 \mathrm{BCP}$ are expected to also affect the $\mathrm{C} 2-\mathrm{O} 5-\mathrm{H} 6$ group undergoing a bending vibration, which is strongly coupled to the methylene group wagging for $\mathrm{HH}$ species, but is expected to be uncoupled for HT. Our analysis therefore, can be used for the interpretation of infrared (IR) spectra to provide explanations of classical mass-dependent isotopic shifts as well as modifications in mode coupling and intensity changes.

The feasibility however, of such studies is still very limited, not only due to expected very weak chiroptical signals but even creation of alpha carbon monosubstitued $\mathrm{D} / \mathrm{T}$ glycine in suitable amounts. As a result, theoretical analysis with NG-QTAIM at present is a unique highly sensitive method for the detection of isotopic chirality in glycine, an amino acid that is present in meteoritic organic compounds.

Furthermore this analysis demonstrates the possibility of directly relating the $\mathbf{P}_{\boldsymbol{\sigma}} / \boldsymbol{\Sigma}_{\boldsymbol{\sigma}}$ chirality to the specific $\mathrm{D} / \mathrm{T}$ isotopic substitution for a molecule in natural conditions, without the need to introduce external electric fields or perform challenging and difficult to interpret spectroscopic experiments. In future, experiments such as those undertaken by Beaulieuet al. on neutral molecules ${ }^{28}$ could be undertaken to detect coherent helical motion of bound electrons of formally achiral glycine and therefore assign $\mathbf{P}_{\sigma} / \boldsymbol{\Sigma}_{\boldsymbol{\sigma}}$ chirality, possibly including the D isotope of glycine.

Funding: This research was funded by the National Natural Science Foundation of China grant number: 21673071. The One Hundred Talents Foundation of Hunan Province is also gratefully acknowledged for their support of S.J. and S.R.K.

\section{References}

1. R. R. Ernst, Angew. Chem. , $1992,104,817-836$.

2. R. R. Ernst, Angew. Chem. Int. Ed. Engl. , $1992,31,805-823$.

3. N. Prantzos, Nature, 2016 , 529, 33-34.

4. A. D. McNaught and A. Wilkinson, Eds., IUPAC Compendium of Chemical Terminology, 2nd ed. (the 'Gold Book'). , Blackwell Scientific Publications, Oxford, 2nd edition, updated online version created by S. J. Chalk (2019) https://doi.org/10.1351/goldbook., 1997 .

5. M. Quack, Mol. Phys., 1977, 34, 477-504. 
6. I. A. Kashaltov and S. J. Eyles, in Mass Spectrometry in Structural Biology and Biophysics: Architecture, Dynamics, and Interaction of Biomolecules, John Wiley \& Sons, Ltd, 2nd edn.,2012 , pp. 89-126.

7. M. Sattler and S. W. Fesik, Structure , 1996 , 4, 1245-1249.

8. C. Puzzarini and M. Biczysko, in Structure Elucidation in Organic Chemistry, eds. M. Cid and J. Bravo, John Wiley \& Sons, Ltd,2015, pp. 27-64.

9. I. Kaltashov and S. Eyles, 2012, pp. 89-126.

10. L. Ma and M. C Fitzgerald, Chem. Biol. , 2004, 10, 1205-13.

11. D. Balasubramaniam and E. A. Komives, Biochim. Biophys. Acta BBA - Proteins Proteomics , 2013 , 1834, 1202-1209.

12. K. D. Rand, M. Zehl and T. J. Jørgensen, Acc. Chem. Res. ,2014 , 47, 3018-3027.

13. M. Sattler and S. W. Fesik, Structure, 1996 , 4, 1245-1249.

14. D. Marion, Mol. Cell. Proteomics MCP , 2013 , 12, 3006-3025.

15. Y. Sawama, Y. Yabe, H. Iwata, Y. Fujiwara, Y. Monguchi and H. Sajiki, Chem. Weinh. Bergstr. Ger. , 2012, 18, 16436-16442.

16. R. Berger, G. Laubender, M. Quack, A. Sieben, J. Stohner and M. Willeke, Angew. Chem. Int. Ed. , 2005, 44, 3623-3626.

17. D. P. Glavin, A. S. Burton, J. E. Elsila, J. C. Aponte and J. P. Dworkin, Chem. Rev. , 2020 , 120, 4660-4689.

18. B. A. McGuire, P. B. Carroll, R. A. Loomis, I. A. Finneran, P. R. Jewell, A. J. Remijan and G. A. Blake, Science, 2016 , 352, 1449-1452.

19. Y. Aikawa, K. Furuya, U. Hincelin and E. Herbst, Astrophys. J. , 2018 , 855.

20. Y. Oba, N. Watanabe, Y. Osamura and A. Kouchi, Chem. Phys. Lett. , 2015 , 634, 53-59.

21. T. Kawasaki, M. Shimizu, D. Nishiyama, M. Ito, H. Ozawa and K. Soai,Chem. Commun. Camb. Engl. , 2009 , 4396-4398.

22. L. Bartell, E. Roth, C. Hollowell, K. Kuchitsu and J. Jr,Chem. Phys. - CHEM PHYS , 1965 , 42, 2683-2686.

23. L. Bartell and R. Roskos, J. Chem. Phys. , 1966 , 44, 457-463.

24. S. Pizzarello and Y. Huang, Geochim. Cosmochim. Acta ,2005 , 69, 599-605.

25. M. M. Green, J.-W. Park, T. Sato, A. Teramoto, S. Lifson, R. L. B. Selinger and J. V. Selinger, Angew. Chem. Int. Ed. ,1999, 38, 3138-3154.

26. T. Xu, J. H. Li, R. Momen, W. J. Huang, S. R. Kirk, Y. Shigeta and S. Jenkins, J. Am. Chem. Soc. , $2019,141,5497-5503$.

27. T. Xu, X. Nie, S. Li, Y. Yang, H. Früchtl, T. van Mourik, S. R. Kirk, M. J. Paterson, Y. Shigeta and S. Jenkins, ChemPhysChem, 2021 , in press.

28. S. Beaulieu, A. Comby, D. Descamps, B. Fabre, G. A. Garcia, R. Géneaux, A. G. Harvey, F. Légaré, Z. Mašín, L. Nahon, A. F. Ordonez, S. Petit, B. Pons, Y. Mairesse, O. Smirnova and V. Blanchet, Nat. Phys. , 2018, 14, 484-489.

29. N. Weinberg and K. Mislow, Can. J. Chem., 2000 , 78, 41-45. 
30. J. A. Elvidge, in The Multinuclear Approach to NMR Spectroscopy, eds. J. B. Lambert and F. G. Riddell, Springer Netherlands, Dordrecht, 1983 , pp. 169-206.

31. R. F. W. Bader and P. F. Zou, Chem. Phys. Lett. ,1992, 191, 54-58.

32. B. Jiang, J. M. Zuo, N. Jiang, M. O'Keeffe and J. C. H. Spence,Acta Crystallogr. A , 2003 , 59, 341-350.

33. J.-C. Zheng, A. I. Frenkel, L. Wu, J. Hanson, W. Ku, E. S. Božin, S. J. L. Billinge and Y. Zhu, Phys. Rev. B , 2010, 81, 144-203.

34. R. F. W. Bader, Atoms in Molecules: A Quantum Theory, Oxford University Press, USA, New York, 1994 .

35. T. Tian, T. Xu, S. R. Kirk, I. T. Rongde, Y. B. Tan, S. Manzhos, Y. Shigeta and S. Jenkins, Phys. Chem. Chem. Phys., $2020,22,2509-2520$.

36. G. Bazso, G. Magyarfalvi and G. Tarczay, J. Mol. Struct. ,2012 , 1025, 33-42.

37. V. Barone, M. Biczysko, J. Bloino and C. Puzzarini, Phys. Chem. Chem. Phys., 2013, 15, 1358-1363.

38. V. Barone, S. Alessandrini, M. Biczysko, J. R. Cheeseman, D. C. Clary, A. B. McCoy, R. J. DiRisio, F. Neese, M. Melosso and C. Puzzarini, Nat. Rev. Methods Primer, 2021 , 1, 1-27.

39. M. X. Hu, T. Xu, R. Momen, A. Azizi, S. R. Kirk and S. Jenkins, Chem. Phys. Lett. , 2017 , 677, $156-161$.

40. W. J. Huang, T. Xu, S. R. Kirk, M. Filatov and S. Jenkins,Chem. Phys. Lett. , 2018 , 713, 125-131.

41. J. H. Li, W. J. Huang, T. Xu, S. R. Kirk and S. Jenkins, Int. J. Quantum Chem. , 2018 , 119, e25847.

42. H. Guo, A. Morales-Bayuelo, T. Xu, R. Momen, L. Wang, P. Yang, S. R. Kirk and S. Jenkins, J. Comput. Chem., 2016 , 37, 2722-2733.

43. P. Yang, T. Xu, R. Momen, A. Azizi, S. R. Kirk and S. Jenkins,Int. J. Quantum Chem. , 2018, 118, e25565.

44. T. Xu, L. Wang, Y. Ping, T. van Mourik, H. Früchtl, S. R. Kirk and S. Jenkins, Int. J. Quantum Chem. , 2018,118, e25676.

45. T. Xu, J. Farrell, R. Momen, A. Azizi, S. R. Kirk, S. Jenkins and D. J. Wales, Chem. Phys. Lett., 2017 , 667, 25-31.

46. T. Xu, J. H. Li, R. Momen, W. J. Huang, S. R. Kirk, Y. Shigeta and S. Jenkins, J. Am. Chem. Soc. , 2019, 141, 5497-5503.

47. N. C. Handy, Y. Yamaguchi and H. F. Schaefer, J. Chem. Phys. ,1986 , 84, 4481-4484.

48. W. Kutzelnigg, Mol. Phys. , 1997, 90, 909-916.

49. D. A. Matthews, L. Cheng, M. E. Harding, F. Lipparini, S. Stopkowicz, T.-C. Jagau, P. G. Szalay, J. Gauss and J. F. Stanton, J. Chem. Phys., 2020 , 152, 214108.

50. Michael J. Frisch, G. W. Trucks, H. Bernhard Schlegel, Gustavo E. Scuseria, Michael A. Robb, James R. Cheeseman, Giovanni Scalmani, Vincenzo Barone, Benedetta Mennucci, G. A. Petersson, H. Nakatsuji, M. Caricato, Xiaosong Li, H. P. Hratchian, Artur F. Izmaylov, Julien Bloino, G. Zheng, J. L. Sonnenberg, M. Hada, M. Ehara, K. Toyota, R. Fukuda, J. Hasegawa, M. Ishida, T. Nakajima, Y. Honda, O. Kitao, H. Nakai, T. Vreven, J. A. Montgomery Jr., J. E. Peralta, François Ogliaro, Michael J. Bearpark, Jochen Heyd, E. N. Brothers, K. N. Kudin, V. N. Staroverov, Rika Kobayashi, J. Normand, Krishnan Raghavachari, Alistair P. Rendell, J. C. Burant, S. S. Iyengar, Jacopo Tomasi, M. Cossi, N. Rega, N. J. Millam, M. Klene, J. E. Knox, J. B. Cross, V. Bakken, C. Adamo, J. Jaramillo, R. Gomperts, R. E. Stratmann, O. Yazyev, A. J. Austin, R. Cammi, C. Pomelli, J. W. Ochterski, R. L. Martin, K. Morokuma, V. G. Zakrzewski, G. A. Voth, P. Salvador, 
J. J. Dannenberg, S. Dapprich, A. D. Daniels, Ö. Farkas, J. B. Foresman, J. V. Ortiz, J. Cioslowski and D. J. Fox, Gaussian 09, Revision E.01 , Gaussian, Inc., 340 Quinnipiac St Bldg 40 Wallingford, CT 06492 USA,2009 .

51. T. A. Keith, AIMAll, Revision 19.10.12, TK Gristmill Software, Overland Park KS, USA, 2019.

52. S. R. Kirk, srk/AIMPAC2-Suite, 2020 . 